\title{
VARIACIÓN ESPACIO-TEMPORAL DE LOS PROCESOS HIDROLÓGICOS DEL SUELO EN VIÑEDOS CON ELEVADAS PENDIENTES (VALLE DEL RUWER-MOSELA, ALEMANIA)
}

\author{
J. RODRIGO-COMINO ${ }^{1,2^{*}}$, M. SEEGER ${ }^{1}$, J.M. SENCIALES ${ }^{2}$, \\ J.D. RUIZ-SINOGA ${ }^{2}$, J.B. RIES ${ }^{1}$
}

${ }^{1}$ Departamento de Geografía Física, Universidad de Trier, Alemania. ${ }^{2}$ Departamento de Geografía, Universidad de Málaga, España.

\begin{abstract}
RESUMEN. Los suelos de los viñedos del valle del Ruwer-Mosela (Alemania) cultivados en elevadas pendientes muestran una alta variabilidad espacio-temporal en su dinámica hidrológica. A través del uso del permeámetro de Guelph se realizaron un total de cuarenta y dos experimentos en viñedos jóvenes y viejos para medir las tasas de infiltración, la conductividad hidráulica y el potencial del flujo matricial. Los ensayos fueron realizados antes de la vendimia coincidiendo con la primavera y el verano (con el suelo relativamente seco y sin actividad reciente de pisadas y maquinaria), y tras la cosecha en otoño (con un manto edáfico húmedo, con señales de compactación y un menor contenido de materia orgánica).

En general, todos los parámetros analizados fueron mucho más elevados en los viñedos jóvenes que en los viejos y aumentaron tras la vendimia. En las viñas jóvenes los tres parámetros analizados mostraron sus mayores valores en la parte media (398,5 $\mathrm{mm} \mathrm{h}^{-1}$ de tasa de infiltración, 89,2 $\mathrm{mm} \mathrm{h}^{-1}$ de conductividad hidráulica y $62,8 \mathrm{~mm}^{2} \mathrm{~h}^{-1}$ de potencial del flujo matricial). Por su parte, en las viñas viejas se observó un descenso de la infiltración desde la parte superior a la inferior de la parcela (desde 42,5 a 16,8 $\mathrm{mm} \mathrm{h}^{-1}$ ). Los resultados de la conductividad hidráulica y el potencial del flujo matricial marcaron también una dinámica hidrológica parecida: descenso de los parámetros conforme se desciende en la parcela (13,2 a 5,4 $\mathrm{mm} \mathrm{h}^{-1}$ y desde 5,5 a 2,5 $\mathrm{mm}^{2} \mathrm{~h}^{-1}$ ). Por ultimo, se observó cómo el factor que mayor coeficiente de determinación obtuvo con estas dinámicas hidrológicas fue la variación del contenido de agua en el suelo y las prácticas agrícolas.
\end{abstract}

Spatial and temporal variation of soil hydrological processes on steep slope vineyards (Ruwel-Mosel Valley, Gemany)

ABSTRACT. The vineyards of Ruwer-Mosel valley (Germany) cultivated on steep slopes showed a high spatial and temporal variability of hydrological dynamics. Forty two experiments were carried out using a Guelph permeameter in old and young vines to measure the infiltration rates, the hydraulic conductivity and the 
soil matrix flux potential. The essays were performed before (spring-summer) and after (autumn) the harvest with dry soil conditions and without soil tillage signals, and with humid soil conditions, signals of soil farming (wheel traffic and footprints) and a decrease of organic matter, respectively.

In general, the results of the young vineyards were higher than the values of the old vineyards. Furthermore, all the rates increased after the harvest. For the young vineyards, the most elevated values were registered on the middle slope $\left(398.5 \mathrm{~mm} \mathrm{~h}^{-1}\right.$ infiltration rate, $89.2 \mathrm{~mm} \mathrm{~h}^{-1}$ hydraulic conductivity and $62.8 \mathrm{~mm}^{2} \mathrm{~h}^{-1}$ soil matrix flux potential). The older vines showed a decrease of the infiltration from the upper slope to the foot slope (from 42.5 to $16.8 \mathrm{~mm} \mathrm{~h}^{-1}$ ). Hydraulic conductivity and soil matrix flux potential showed the same hydro-dynamics: from 13.2 to 5.4 $m m h^{-1}$ and from 5.5 to $2.5 \mathrm{~mm}^{2} h^{-1}$, respectively. Finally, it was observed that the most correlated factor with these hydrological processes was the soil moisture content and the soil tillage practices.

Palabras clave: viñedos, permeámetro de Guelph, infiltración, procesos hidrológicos, Valle del Ruwer-Mosela.

Key words: vineyards, Ruwer-Mosel valley, Guelph permeameter, infiltration, hydrologycal processes.

Recibido el 15 de enero de 2016 Aceptado el 9 de marzo de 2016

*Correspondencia: J. Rodrigo-Comino, Departamento de Geografía Física, Universidad de Trier, C.P. Behringstraße, D-54286, Trier, Alemania. E-mail: geo.jrc@gmail.com

\section{Introducción}

Los espacios ocupados por viñedos muestran particularidades en el manejo agrícola y unas condiciones medioambientales identificables de suelo, clima, paisaje y topografía, con influencias directas en las composición de la uva (Fischer et al., 1999; Resolution OIV/VITI 333/2010, 2010; van Leeuwen et al., 2010; Cadot et al., 2012). Actualmente, numerosos autores evidencian en sus estudios la importancia de los procesos de degradación que han sucedido en estas áreas durante décadas, inducidas por la implementación en los usos tradicionales del suelo de maquinaria pesada (tractores, cosechadoras...), de nuevos productos químicos (fertilizantes, herbicidas...) y por la intensificación de la producción (Arnáez et al., 2007; Martínez-Casasnovas et al., 2009; Bruggisser et al., 2010; López-Piñeiro et al., 2013; Salome et al., 2014; Likar et al., 2015). Todas estas actividades están afectando notablemente a uno de los componentes más importantes de los viñedos: el suelo.

Por este motivo, en la ciencia del suelo y en la Geomorfología están aumentando los estudios que tienen como objeto detectar los flujos hídricos que activan las tasas de erosión y la transferencia de productos contaminantes a lo largo de las laderas (Costantini et al., 2015; Lieskovský and Kenderessy, 2014; Morvan et al., 2014; Novara et al., 2011; Tagues et al., 2015; Prosdocimi et al., 2016a). En esta línea, numerosos autores han confirmado la importancia 
de los componentes superficiales y sub-superficiales del suelo, como elemento condicionante de todas estas dinámicas hidrológicas (Corbane et al., 2012; Kosmas et al., 1997; Ramos y Martínez-Casasnovas, 2006; Ruiz Sinoga y Martínez Murillo, 2009). Sin embargo, son menores las referencias en estos estudios al desplazamiento del agua a lo largo de la ladera en forma de escorrentía sub-superficial tras la infiltración o percolación y sus factores condicionantes. Sin duda, en áreas como el valle del Mosela con unas tasas de infiltración elevadas (Rodrigo Comino et al., 2015a, 2015b, 2016) cuestiones sobre la dirección del flujo sub-superficial, la variabilidad temporal de la intensidad o los factores que la condicionan deberían ser atendidas con premura si se pretende estudiar en profundidad para dicho medio su completa dinámica eco-geomorfológica. En estudios realizados en España, Italia o Francia, ha quedado demostrado que en pocas hectáreas pueden confluir numerosos procesos que, detectados y cuantificados correctamente, pueden aportar información útil al agricultor para diseñar estrategias sostenibles para su cultivo (Ortigosa Izquierdo y Lasanta Martínez, 1984; Leonard y Andrieux, 1998; Ramos y Martínez-Casasnovas, 2006; Corbane et al., 2012; Biddoccu et al., 2013).

Los diferentes recorridos que el agua realiza, a través del sistema de poros y fracturas de los agregados del suelo, puede conllevar procesos tan variados como el transporte de solutos, iluviación y eluviación o lavado de materiales finos, infiltración-percolación, escorrentía superficial o sub-superficial, pseudogleyzación y/o gleyzación y variación de la cantidad de agua disponible para las plantas tras la lluvia (Wu et al., 1992; Price et al., 2010; Archer et al., 2013; Bodner et al., 2013; Jirkû et al., 2013).

Para analizar estas dinámicas hidrológicas del suelo, sería de vital importancia conocer tres de las propiedades físicas de los suelos más relevantes ligadas a estas dinámicas: la infiltración, la conductividad hidráulica $\left(\mathrm{Kf}_{\mathrm{s}}\right)$ y el potencial del flujo matricial $(\Phi)$.

Existen tres tipos de mediciones de estos parámetros, tanto en laboratorio como directamente en el campo. Los primeros métodos se apoyan en resultados obtenidos con programas informáticos, que permiten generar modelos a partir de situaciones reales aportando datos sobre humedad, materia orgánica, textura o densidad aparente; sin embargo, son ampliamente discutidos (Kumar et al., 2010; Bodner et al., 2013; Rienzner y Gandolfi, 2014). Los otros dos métodos se nutren de los datos obtenidos in situ con el análisis de las muestras recogidas en el campo sin perturbar o directamente con instrumentos de campo como los infiltrómetros de mini-disco o de doble anillo (Cerdà, 1997; Peter y Ries, 2013). No obstante, también reciben numerosas críticas debido al tiempo invertido durante los experimentos o el elevado número de repeticiones que deben realizarse para observar una tendencia clara. En este sentido, para áreas de estudio como los viñedos, que suelen estar cultivados en laderas con pendientes pronunciadas y con una elevada pedregosidad, probablemente el instrumento más útil para medir la permeabilidad, la transmisividad, la infiltración o la conductividad hidráulica sea el permeámetro de Guelph (GP). Su funcionalidad ha sido probada y comparada con otros instrumentos, tales como el simulador de lluvia o los infiltrómetros en numerosas ocasiones (Wu et al., 1992; Gupta et al., 1993, 2006; Kodešová et al., 2010; Gwenzi et al., 2011; MacDonald et al., 2012; Archer et al., 2013; Bagarello et al., 2014; Jačka et al., 2014; Huang et al., 2014, 2016). Su funcionamiento se rige a un principio modificado de la botella de Mariotte, el cual envía una constante descarga de agua que actúa tridimensionalmente en forma de columna vertical, que actúa 
dentro de un orificio cilíndrico en el suelo (Elrick y Reynolds, 1992; Reynolds y Elrick, 2002; Reynolds y Lewis, 2012).

Los dos objetivos principales de este estudio fueron conocer cuál fue la velocidad de cambio y la respuesta hidrológica del suelo según la edad del viñedo, el sector de la ladera y la época del año. Este estudio se llevó a cabo en dos parcelas experimentales cultivadas con vides de diferentes edades ( 3 y 35-40 años, respectivamente) que poseen distintas características edafológicas (granulometría, profundidad, materia orgánica...) y que se sitúan sobre elevadas pendientes. Con este fin, se procedió también a esclarecer otras cuestiones como: i) determinar el tipo de suelo y las diferencias de sus propiedades a lo largo de la ladera; ii) calcular las tasas de infiltración, la conductividad hidráulica y el potencial del flujo matricial; iii) observar si existen diferencias especiales en el comportamiento hidrológico del suelo (zona alta, media y baja de la ladera) y temporales (antes -verano- y después -otoño- de la vendimia); y iv) detectar si algún parámetro medido conjuntamente para cada experimento (pendiente, materia orgánica o humedad) está relacionado o no con dichos cambios antes y después de la vendimia.

\section{2. Área de estudio}

El área de estudio se localiza cerca de una pequeña localidad llamada Waldrach (Fig. 1), situada en el valle del Ruwer, un afluente del río Mosela en el oeste de Alemania (49.7418N; 6.7524E; Trier, Renania-Palatinado). La precipitación anual ronda los $765 \mathrm{~mm}$ y los máximos pluviométricos mensuales se concentran en verano (65-72 $\mathrm{mm})$. La temperatura media anual es de $9,3^{\circ} \mathrm{C}$ con máximas en verano $\left(16,2-17,6^{\circ} \mathrm{C}\right)$ y mínimas con riesgos de heladas en invierno $\left(1,5-2,3^{\circ} \mathrm{C}\right)$. El tipo de clima según la clasificación de Köppen-Geiger (1954) es $C f b$.

El material parental sobre el que se asientan los cultivos está compuesto por una base litológica del Paleozoico no calcárea y con una morfología ondulada con grauwacas devónicas, pizarras y cuarcitas. En algunas ocasiones, se pueden apreciar restos de materiales sedimentarios finos procedentes del curso fluvial del Ruwer datados del Pleistoceno (Richter, 1975, 1980).

El trabajo se llevó a cabo en un campo cultivado de vides con elevadas pendientes (20$30^{\circ}$ ) entre 200 y 250 m.s.n.m. con una exposición SSO. Las dos parcelas estudiadas se sitúan dentro de una geoforma principal (ladera) que consta de hileras de vides con longitudes aproximadas de entre 60 y 65 metros cada una. Se pueden diferenciar dos tipos de viñedos en cada parcela según su edad de plantación. El primero posee una edad aproximada de entre 35 a 40 años y el segundo menos de 3 años. Los más jóvenes antes de adquirir este nuevo uso agrícola estaban clasificados como tierras abandonadas, que también habían sido cultivadas con vides con anterioridad. Previas a la nueva plantación, el suelo fue removido con maquinaria pesada, con objeto de airear los horizontes más profundos y eliminar las raíces de la antigua vegetación de tipo matorral que había colonizado dicho espacio.

Ambas áreas se organizan con un marco de plantación de 0,9x1 m y están cultivadas con la misma variedad de uva blanca: Riesling. 


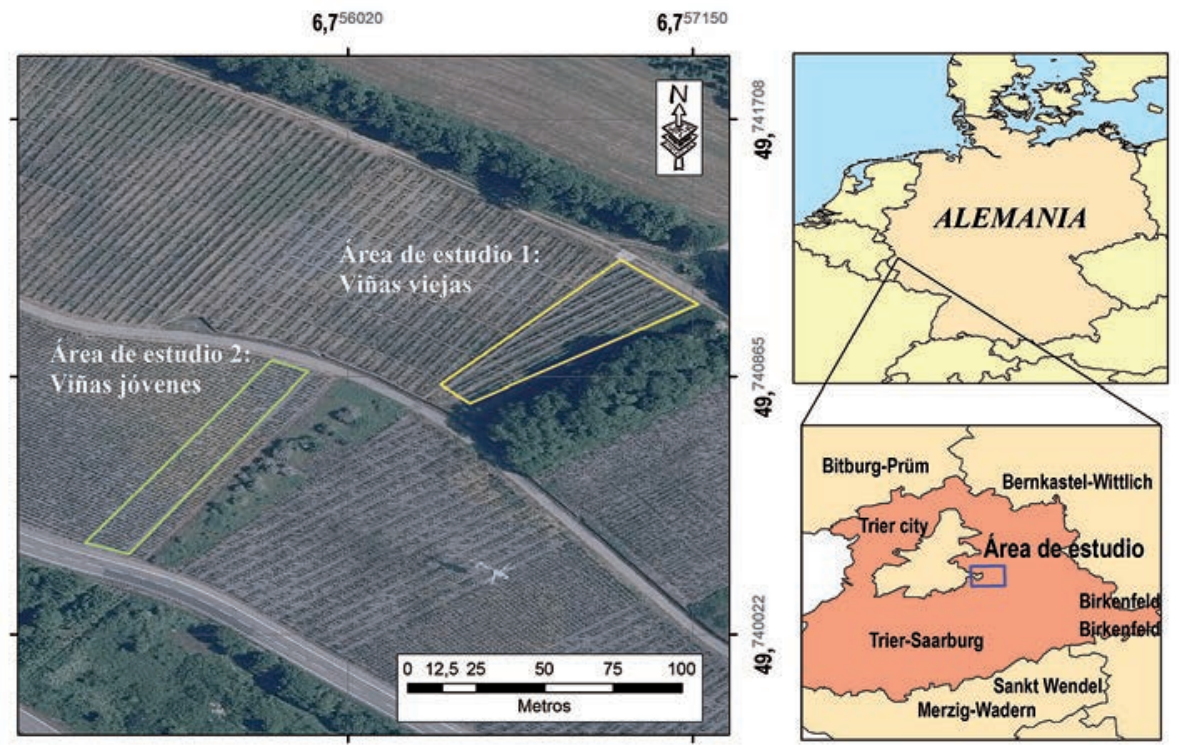

Figura 1. Localización del área de estudio.

Se aprecian notables diferencias en el cubrimiento vegetal aéreo en cada viñedo, siendo la cobertura foliar mucho más densa en las viñas viejas que en las jóvenes a partir del comienzo del periodo de brotación. La cobertura radicular también abarca más superficie efectiva en las viñas viejas y muestran mayor grosor. También los tamaños de los troncos en las viñas viejas son de una mayor anchura y altura desde el callo hasta la parte donde comienzan a salir las yemas de los nuevos brotes. Por último, se registran mayores pendientes y una mayor cantidad de lascas pizarrosas bajo las plantas en los viñedos jóvenes que en los viejos.

En ambos espacios cultivados, el manejo del suelo está ligado a: i) una remoción de los horizontes de entre 15 y $20 \mathrm{~cm}$ de espesor con un tractor que desciende perpendicularmente a las curvas de nivel antes (abril y mayo) y después (finales de octubre y principios de noviembre) de la vendimia; ii) un mantenimiento de la cobertura vegetal herbácea entre calles y bajo las cepas que llega a alcanzar en verano los 20 centímetros de altura; iii) la colocación de cantos de pizarra bajo las vides con objeto de conservar el calor emitido por la radiación solar y proteger el suelo frente al efecto del golpeo de las gotas de agua durante las tormentas; iv) un tipo de plantación en espaldera para favorecer al máximo los procesos fotosintéticos y la asimilación de azúcar; v) uso de herbicidas y fitosanitarios a través de vuelos con helicóptero; vi) recogida de la uva a mano, utilizando gran cantidad de mano de obra durante la segunda mitad de octubre y primera de noviembre que deja severas marcas en forma de pisadas a lo largo de la ladera.

Como consecuencia de este manejo, se desarrollan procesos erosivos tales como regueros y cárcavas provocados por el uso de maquinaria o el paso de los agricultores, y marcas por la pérdida de suelo en los tocones de las viñas han sido detectadas en recientes estudios (Rodrigo Comino et al., 2015a, 2015b; 2016). 


\section{Métodos}

\subsection{Análisis de suelos y elaboración de perfiles}

Las muestras de suelos fueron recogidas entre noviembre y diciembre de 2014. El muestreo se llevó a cabo en las dos parcelas con plantas de diferentes edades en tres posiciones distintas (alta, media y baja) (Fig. 2), a dos profundidades $(0-5 \mathrm{~cm}$ y $5-15 \mathrm{~cm}$ ) y entre las calles. Todas ellas fueron recogidas con tres repeticiones cada una.

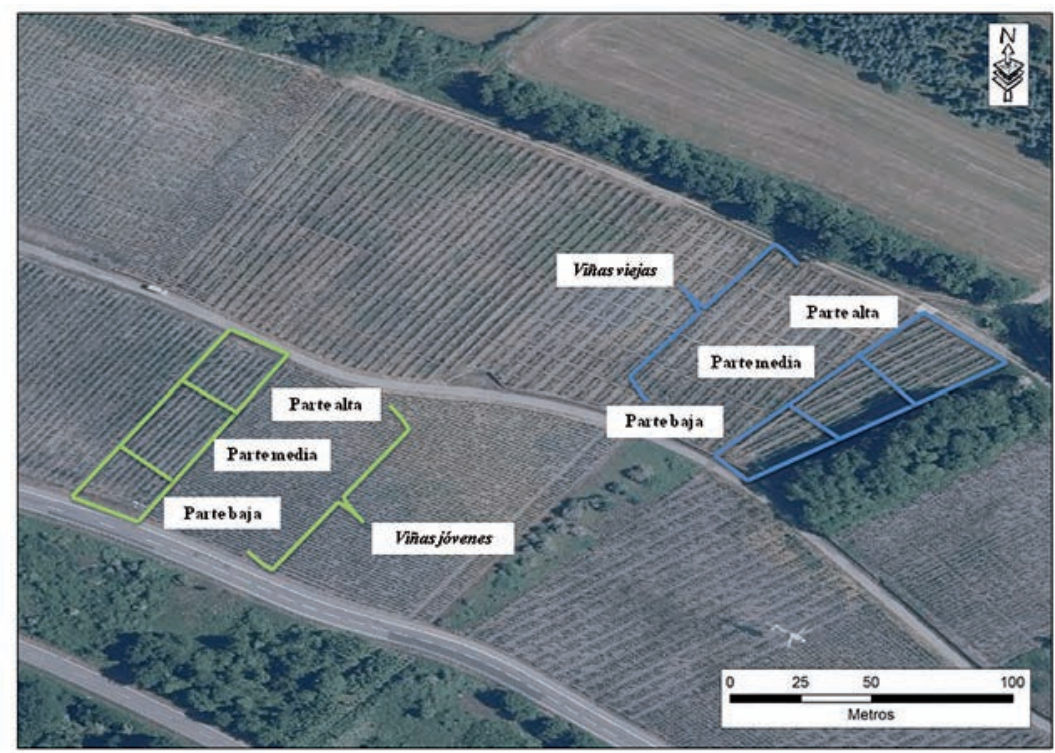

Figura 2. Distribución del muestreo de suelos en la geoforma principal (ladera) y sobre las parcelas de viñas viejas y jóvenes.

Un total de 18 muestras de entre 3 y 4 kilos fueron secadas en el laboratorio a temperatura ambiente y tamizadas las partículas menores a $2 \mathrm{~mm}$. A partir de aquí, diferentes parámetros químicos y físicos fueron analizados.

En primer lugar, la textura fue medida utilizando un Coulter LS230, capaz de medir, combinando diferentes patrones de difracción de los haces de luz, el tamaño de partículas entre $0,004 \mu \mathrm{m}$ y $2000 \mu \mathrm{m}$. También, se calcularon los resultados del total del contenido de carbono orgánico, utilizando un horno mufla aplicando $105^{\circ} \mathrm{C} \mathrm{y} 430^{\circ} \mathrm{C}$ para quemar las partículas (Davies, 1974; Rosell et al., 2001).

Además, se midió la conductividad eléctrica con un conductímetro, el contenido en carbonatos con un calcímetro de Bernard y el pH utilizando $\mathrm{KCl}$ y agua destilada (1:5). Diferencias mayores que uno entre el $\mathrm{pH}$ analizado con esas dos sustancias indicar la tendencia a la acidez de las muestras (Porta et al., 2014). Todos estos parámetros químicos han sido analizados teniendo en cuenta que poseen una gran capacidad indicativa sobre otras propiedades edáficas tales como la salinidad, la mineralogía o la capacidad de intercambio catiónico (Gruber y Kosegarten, 2002; Lesch y Corwin, 2003; Taylor et al., 
2009; Jackson, 2014). Finalmente, se describieron tres perfiles de suelos a las mismas alturas de la ladera que las muestras recogidas, utilizando la metodología de FAO-WRB (IUSS Working Group WRB, 2006; 2007; 2014).

\subsection{Medición de la infiltración, la conductividad hidráulica saturada y el potencial del flujo matricial del suelo en campo}

El permeámetro de Guelph (Fig. 3) fue utilizado tras diseñar un protocolo de uso según los requerimientos del área de estudio (usos del suelo, elevadas pendientes, alta pedregosidad...) y recopilando diversas experiencias de otras investigaciones (Bagarello, 1997; Zhang et al., 1998; Soil moisture Equipment Corp, 2008; Kodešová et al., 2010; MacDonald et al., 2012; Reynolds y Lewis, 2012): i) se ha realizado un total de 42 experimentos de permeabilidad, correspondiendo con los lugares de las muestras recogidas, durante la época anterior (mayo-septiembre) y posterior a la vendimia (noviembre-diciembre); ii) dos experimentos se realizaron en el mismo orificio (entre 10 y $25 \mathrm{~cm}$ de profundidad y 4 y $10 \mathrm{~cm}$ de radio), uno con 5 y otro con $10 \mathrm{~cm}$ de espacio entre el extremo del cilindro del permeámetro y la columna de agua constante sobre el suelo; iii) la duración del experimento fue de 30 minutos cada uno o cuando el reservorio principal que contenía el agua se vaciaba; iv) la observación de datos se llevó a cabo en intervalos de dos minutos medidos con un cronómetro; v) el steady rate o constante fue obtenido a partir del promedio de todas las mediciones del experimento; iv) fueron desechados los experimentos donde el permeámetro se vació en menos de dos minutos o el orificio creado se desbordó de agua a causa de una rápida saturación.
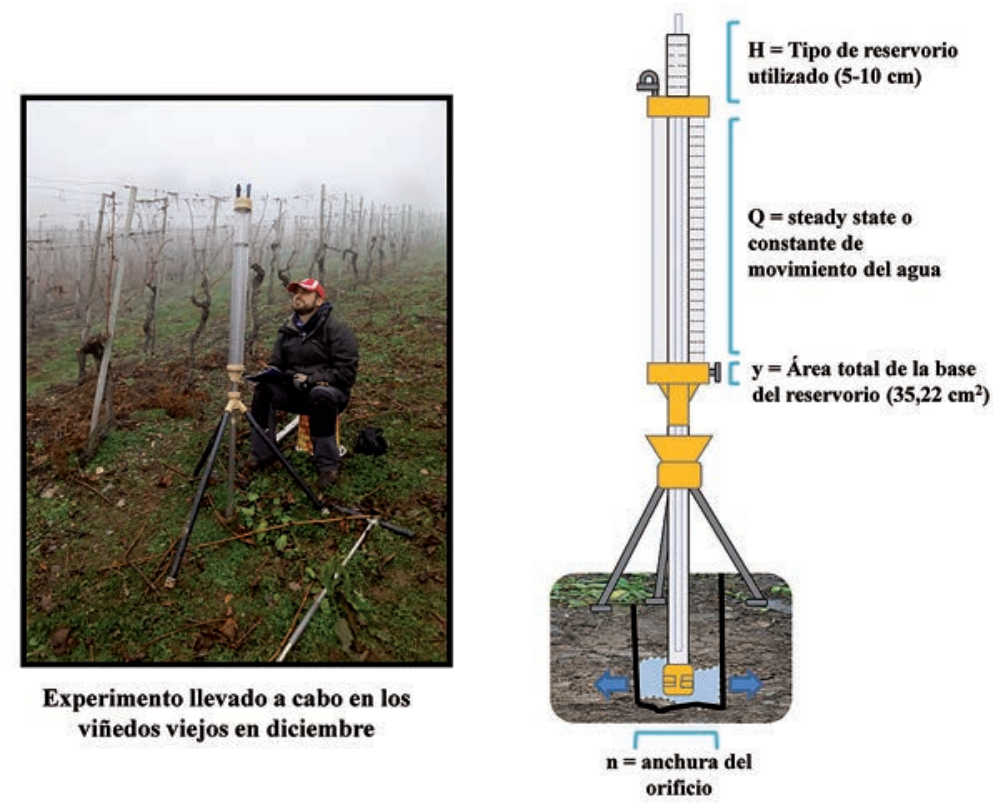

Figura 3. Ejemplo de experimento llevado a cabo durante el trabajo de campo y croquis del permeámetro de Guelph. 
Tras obtener las constantes (o steady rates), se desarrolló una ecuación para el cálculo de la infiltración y se aplicaron dos funciones de pedo-transferencia ya aplicadas en numerosos estudios con objeto de que los resultados puedan ser comparados con otras áreas (Reynolds, 1986; Zhang et al., 1998; Reynolds y Elrick, 2002):

$$
\begin{aligned}
& \text { Tasa de infiltración }=\left(\frac{\frac{\mathrm{y} * \mathrm{Q}_{1}}{1000}}{2 \pi(\mathrm{a} * \mathrm{H} 1)}\right) * 60\left(\mathrm{~mm} \mathrm{~h}^{-1}\right) \\
& \left.\mathrm{K}_{\mathrm{fs}} \text { (conductividad hidráulica saturada }\right)=\left(\frac{\mathrm{C} 1 * \mathrm{Q} 1}{2 \pi \mathrm{H}^{2}+\pi \mathrm{a}^{2} \mathrm{C} 1+2 \pi\left(\frac{\mathrm{H} 1}{\alpha}\right)}\right) * 600\left(\mathrm{~mm} \mathrm{~h}^{-1}\right) \text { (2) } \\
& \Phi \text { potencial del flujo matricial }=\left(\frac{\mathrm{C} 1 * \mathrm{Q} 1}{\left(2 \pi \mathrm{H}^{2}+\pi \mathrm{a}^{2} \mathrm{C} 1\right) \alpha+2 \pi(\mathrm{H} 1)}\right) * 6000\left(\mathrm{~mm}^{2} \mathrm{~h}^{-1}\right) \\
& \mathrm{C} 1 \text { = factor de la forma del orificio } \\
& \mathrm{y}=\text { área total de la base del reservorio principal del permeámetro }\left(35,22 \mathrm{~cm}^{2}\right) \\
& \mathrm{Q} 1=\text { steady rate o constante del movimiento del agua desde el permeámetro hacia } \\
& \text { el suelo (cm min } \left.{ }^{-1}\right)
\end{aligned}
$$

$\mathrm{H} 1$ = corresponde al valor del experimento con $5 \mathrm{~cm}$ en la columna constante de agua (H2 se calculará para el experimento con $10 \mathrm{~cm})$.

$\alpha=$ constante calculada a través de una extrapolación de la capilaridad y macrocospicidad del suelo.

$$
\mathrm{a}=\text { radio del orificio }(\mathrm{cm}) \text {. }
$$

Por último, hay que añadir que se recogieron en un radio de 0,5 metros muestras de suelo de entre 500 y 1000 gramos para calcular, con el mismo procedimiento explicado anteriormente (apartado 3.1.), la materia orgánica y la humedad del suelo (secada a $105^{\circ} \mathrm{C}$ en una estufa). Además se anotaron los grados de la pendiente de la parte superficial del orificio con un clinómetro.

\section{Resultados}

\subsection{Perfiles edáficos y análisis de suelo}

En primer lugar se presenta una descripción de los perfiles realizados en las dos laderas analizadas (Fig. 4).

Todos los perfiles fueron descritos con unas condiciones meteorológicas similares: parcialmente nubosas y con algunas precipitaciones de carácter leve $(<2 \mathrm{~mm})$. Todos 
tenían signos de labrado en el suelo y se podía oler la reciente fumigación esparcida por las avionetas. En ningún perfil se observaron afloramientos rocosos y en todos se podía observar una cobertura vegetal de herbáceas de no más de $15 \mathrm{~cm}$ de altura. El contenido de humedad en los perfiles de los viñedos viejos osciló entre 9 y $14 \%$ y en los jóvenes entre 1 y $8 \%$. Cabe destacar la elevada pedregosidad (entre 35 y 65\%) registrada en todos los perfiles analizados.

Con respecto a los horizontes, en general, se pudo distinguir con claridad su delimitación desde la superficie hasta la parte más profunda, con unos límites que oscilaron desde lo abrupto $(0-2 \mathrm{~cm})$ a lo gradual $(0-15 \mathrm{~cm})$.

Viñedo antiguo
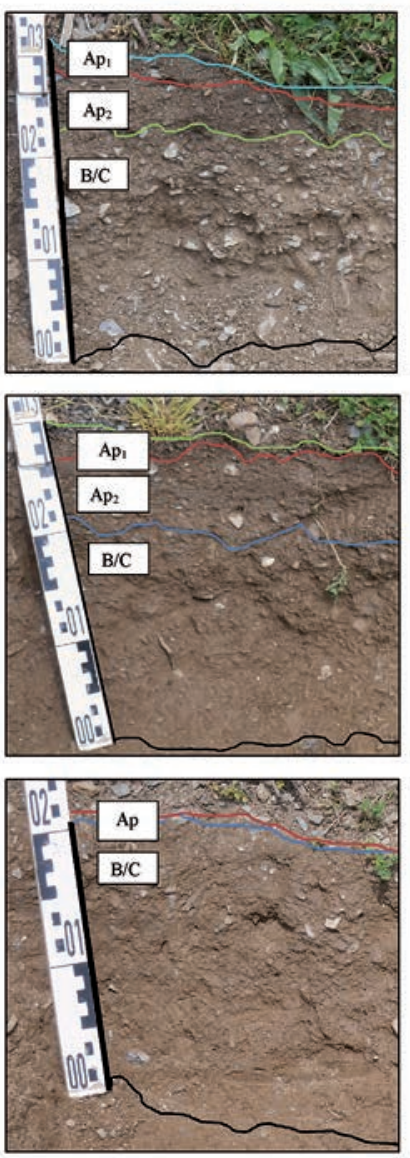

Viñedo joven
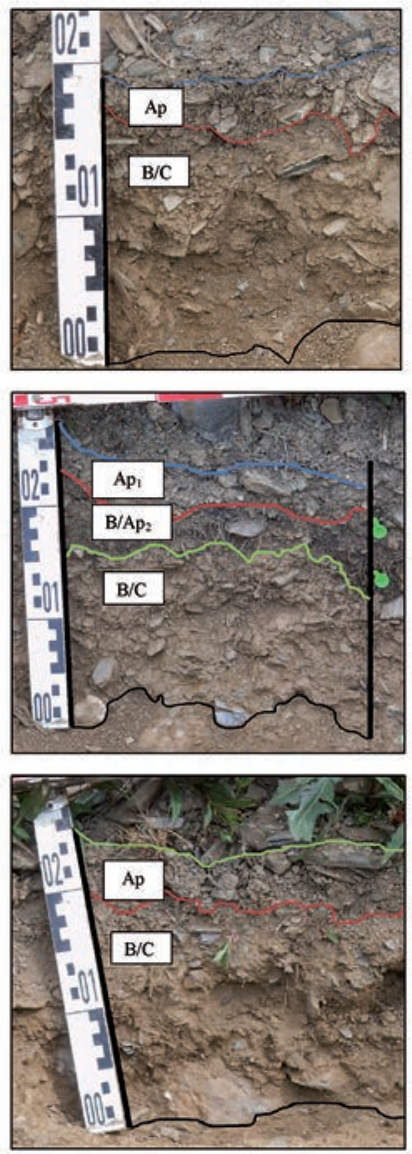

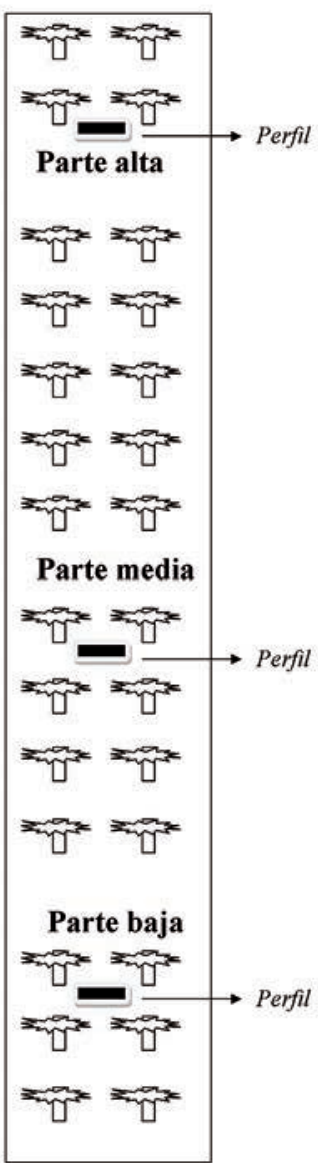

Figura 4. Perfiles realizados en ambas parcelas.

En los viñedos viejos, en la parte superior y media se pudo distinguir un horizonte superficial compuesto por un $\mathrm{Ap}_{1}$ desde 0 a $2 \mathrm{~cm}$ con mayor contenido aparente en materia orgánica, de color oscuro (2.5YR 3-5 en seco y 2.5YR 4-4 en húmedo), sin estructura, 
con una elevada porosidad (15-40\%), muy alterado por la erosión (por la aparición de pequeños surcos y lascas pizarrosas lavadas) y con un elevado contenido de raíces muy finas $(<0,5 \mathrm{~mm})$. A continuación, aparece un $\mathrm{Ap}_{2}$ entre los 7-10 cm con un límite algo más difuso y discontinuo debido a la remoción por el laboreo. El color no varió entre los perfiles (solo en húmedo pasó a 2.5YR 3-3). La estructura fue consistente y en forma de terrón (10-20 mm) y con una elevada porosidad de tipo intersticial (15-40\%). Al humedecerlo se observó su elevada plasticidad y adhesividad. El número de raíces comenzó a ser menor con respecto al horizonte anterior (20-50 raíces por $\mathrm{cm}^{2}$ ), impedidas por la continuidad y compactación de los horizontes debido al paso de la maquinaria.

Por último, apareció un horizonte B/C $(7-10 \mathrm{~cm})$, que se pudo distinguir en la parte más baja de la parcela, no más profundo de 40 centímetros (en el caso del perfil de la parte baja solo hasta $20 \mathrm{~cm}$ ). Los bordes fueron difusos $(>15 \mathrm{~cm})$ y discontinuos. En húmedo mostró una elevada friabilidad, plasticidad y adhesividad. También se observó el mismo color y grado de compactación.

En los viñedos jóvenes los horizontes mantuvieron una apariencia semejante en lo que se refiere al límite de los horizontes, distinguiéndose un horizonte superficial desde los 0 a los 5-6 cm (Ap) y otro hasta los 20-30 cm (B/C). Sin embargo, es necesario realizar algunas matizaciones. En primer lugar, los niveles de pedregosidad fueron más elevados que en los anteriores perfiles y el color había variado (2.5YR 4-7 en seco, 10YR 4-5 en húmedo). También, se observaron moteados oscuros de materia orgánica y azulados de gley en los agregados algo más prismáticos y compactos que en los viñedos viejos. La consistencia también fue algo mayor y el número de fracturas entre agregados conllevó a que la porosidad observada pareciera mayor que en los anteriores casos. Como nota más destacada, en la parte media de ladera se encontró un horizonte orgánico enterrado entre los 7 y $10 \mathrm{~cm}$ (B/Ap o Apb) con un contenido en materia orgánica visiblemente mayor con respecto a los horizontes superior e inferior.

La Tabla 1 muestra los resultados obtenidos tras la analítica de suelos. Los porcentajes más elevados de materiales mayores a $2 \mathrm{~mm}$ están situados en los viñedos jóvenes en la parte media y baja de la parcela (más del 60\%). La pedregosidad, por el contrario, es menor en el viñedo viejo, aunque también es relativamente elevada (superior al 35\%). En relación a la fracción de arenas, encontramos unas cifras que oscilan entre 20 y $40 \%$, siendo el porcentaje más elevado entre $0-5 \mathrm{~cm}$. Ambas parcelas se caracterizan por elevados porcentajes de limos (mayores a 60\%), sobre todo en los horizontes más superficiales $(0-5 \mathrm{~cm})$. Los valores de las arcillas están entre el 6 y el 11\%, siendo más elevados en casi todos los casos a partir de los 5 primeros centímetros de espesor del suelo.

Con respecto a la materia orgánica, aunque los valores varían a lo largo del año, se pudo observar en el momento de la recolección de la uva que en las viñas viejas los valores estaban entre 4,4 y 5,9\% y en las jóvenes entre 3,4 y 13,7\%. En la conductividad eléctrica y los carbonatos no se observaron grandes variaciones, oscilando entre $0,1 \mathrm{dS} \mathrm{m}^{-1}$ y $0,8 \mathrm{dS} \mathrm{m}^{-1}$ e inferiores a $2,1 \%$, respectivamente. Por lo tanto, se deduce que, como cabía esperar en suelos de elevada pendiente y precipitaciones superiores a $700 \mathrm{~mm}$, estos suelos no mostraron problemas de salinidad. 


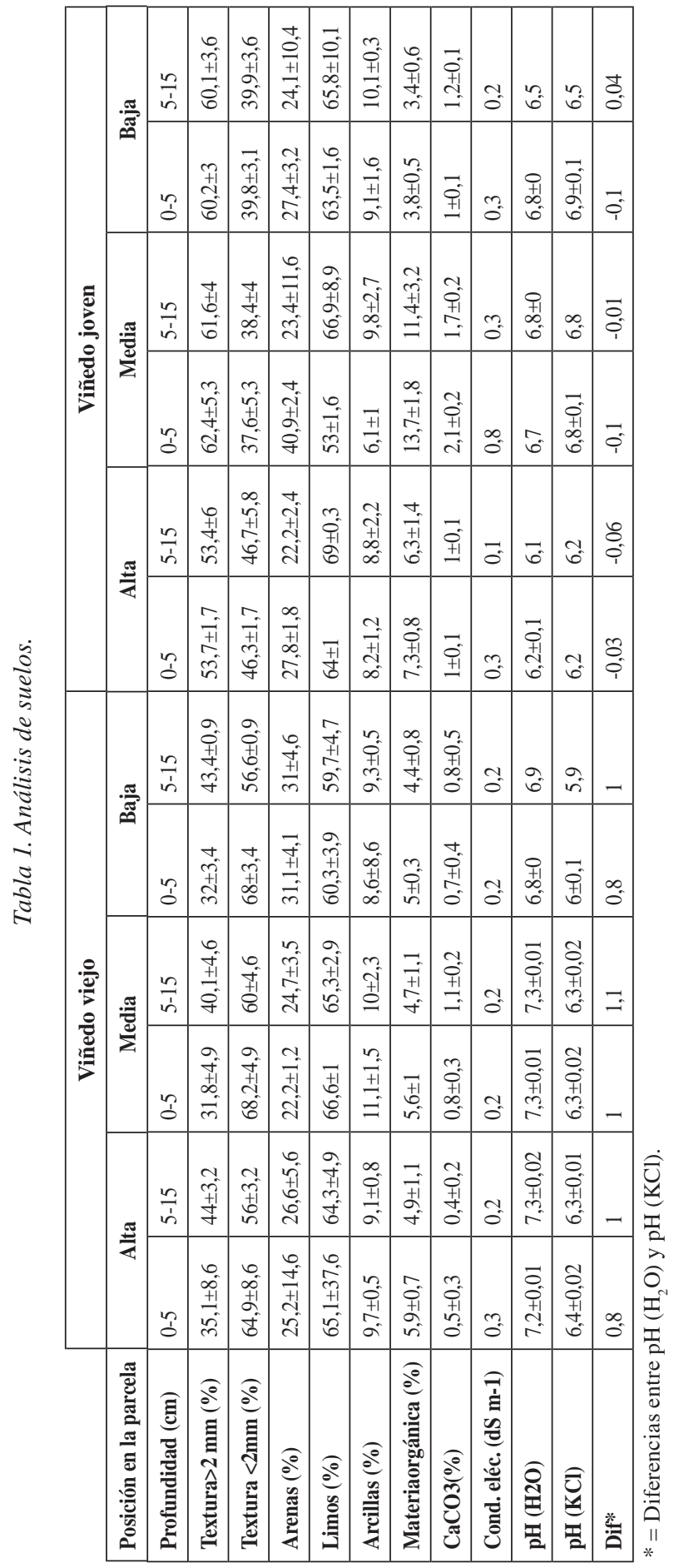


Por último, en lo referido al $\mathrm{pH}$ con $\mathrm{H}_{2} \mathrm{O}$ se pueden apreciar valores en torno a 6,8 y 7,3 para las vides viejas y entre 6,1 y 6,8 para las jóvenes. Al analizar la misma muestra con $\mathrm{KCl}$ y observar la diferencia con el anterior procedimiento, se ha podido distinguir que en los viñedos viejos no existe una tendencia a la acidificación como en los jóvenes, donde todos los valores han mostrado resultados con cifras negativas.

Finalmente, el suelo fue clasificado como Regosol léptico-húmico (IUSS Working Group WRB, 2014), dado su espesor, contenido en materia orgánica y composición textural.

\subsection{Curvas de infiltración obtenidas a partir del Permeámetro de Guelph}

Un total de 42 experimentos fueron llevados a cabo entre las dos áreas de estudio (21 en cada parcela), distribuidos entre la parte alta, media y baja de las parcelas (7 en cada punto), diferenciando los realizados con una columna de agua de 5 y $10 \mathrm{~cm}$, en la época previa y posterior a la vendimia (Tabla 2). Quedaron excluidos para los cálculos finales aquellos experimentos en los que el suelo se saturó y el agua se salió por los límites del área de estudio ( 1 en los jóvenes y 5 en los viejos). También fueron eliminados aquellos donde el volumen de agua total del permeámetro fue absorbido por el suelo en menos de dos minutos ( 3 en los jóvenes), debido a alguna fisura o canal entre los horizontes sub-superficiales generados al realizar el orificio del área de estudio o simplemente por la elevada macroporosidad que evitaba una medición realista.

Tabla 2. Número de experimentos llevados a cabo con el permeámetro de Guelph.

\begin{tabular}{|l|l|l|l|l|}
\hline & \multicolumn{2}{|c|}{ Viñedo joven } & \multicolumn{2}{c|}{ Viñedo viejo } \\
\hline & $5 \mathrm{~cm}$ & $10 \mathrm{~cm}$ & $5 \mathrm{~cm}$ & $10 \mathrm{~cm}$ \\
\hline $\mathrm{n}$ & 21 & 21 & 21 & 21 \\
\hline Parte alta de la parcela & 7 & 7 & 7 & 7 \\
\hline Parte media de la parcela & 7 & 7 & 7 & 7 \\
\hline Parte baja de la parcela & 7 & 7 & 7 & 7 \\
\hline Antes de la vendimia & 12 & 12 & 12 & 12 \\
\hline Después de la vendimia & 9 & 9 & 9 & 9 \\
\hline Saturación del suelo & 1 & 0 & 2 & 3 \\
\hline Experimento con menos de 2 minutos de duración & 0 & 3 & 0 & 0 \\
\hline Total experimentos con éxito & 20 & 18 & 19 & 18 \\
\hline
\end{tabular}

En la figura 5, se presentan las curvas de infiltración utilizando los promedios de la tasas de infiltración $\left(\mathrm{mm} \mathrm{h}^{-1}\right)$ a lo largo de los experimentos llevados a cabo con éxito (durante media hora de duración) según los anteriores criterios con 5 y $10 \mathrm{~cm}$ en la columna de agua. Se pretenden distinguir los resultados de los promedios totales en ambas parcelas, además de su situación, antes y después de la vendimia.

En todos los gráficos se apreció cómo las tasas de infiltración comenzaban a descender desde el principio del experimento hasta su conclusión. Estas cifras fueron 
muy marcadas en los viñedos jóvenes, sobre todo en los promedios totales y antes de la vendimia, donde en los dos primeros minutos del experimento se registraron cifras muy elevadas. En contraposición, se observó que en los viñedos viejos el descenso fue más prolongado y menos inclinado manteniéndose constante hasta los últimos diez minutos, donde el decrecimiento de la tasa fue algo mayor.

Para los promedios generales, se observó que las tasas de infiltración fueron más elevadas en los viñedos jóvenes que en los viejos, siendo también los valores extremos mucho más marcados. Sin embargo, al hacer una distinción temporal en la realización de los experimentos, se observó que esos valores fueron mucho más bajos en los viñedos jóvenes en la época previa a la vendimia que la posterior. Esta situación también se repitió, pero con menores tasas de infiltración para los experimentos llevados a cabo en los viñedos viejos.
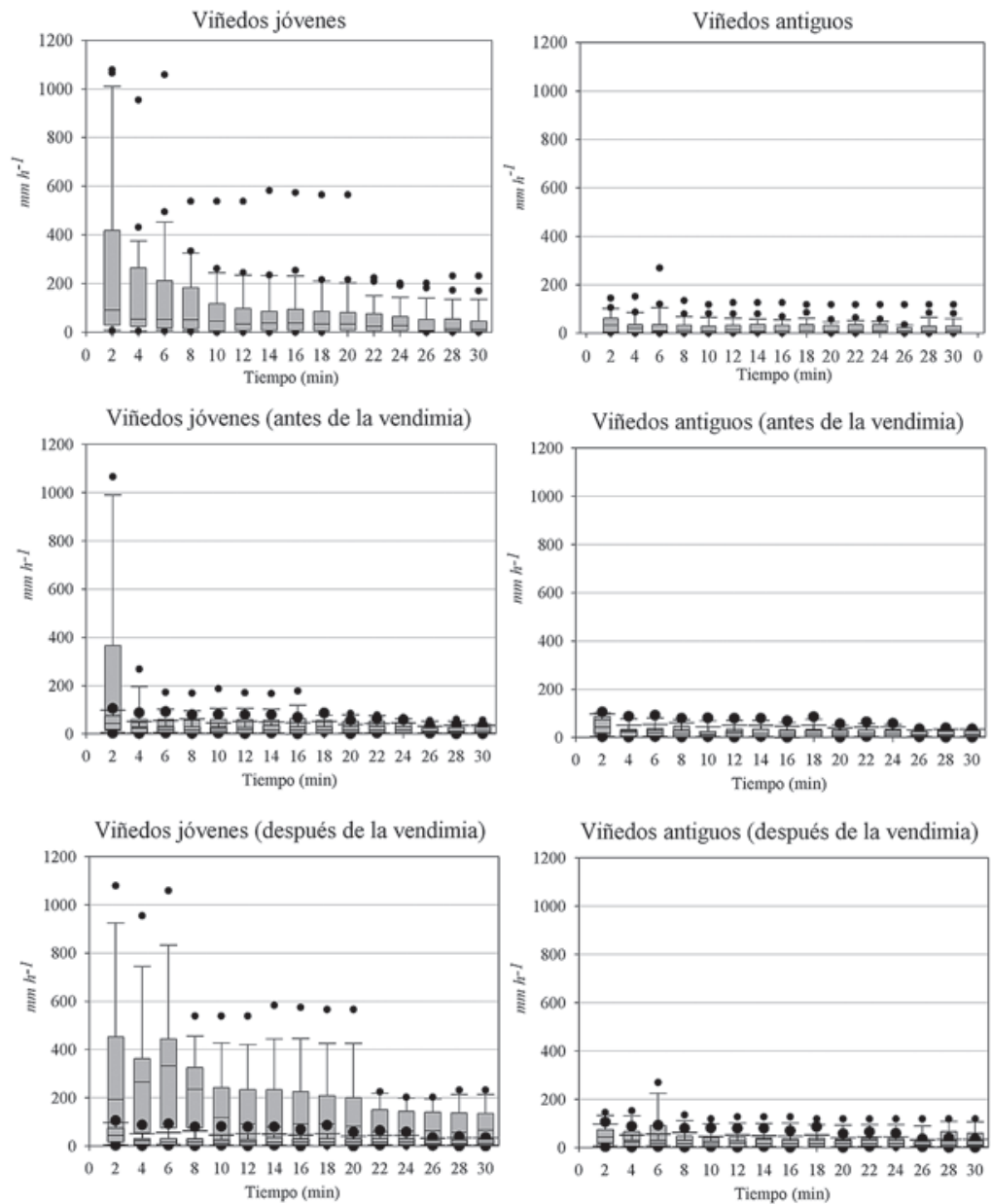

Figura 5. Resultados de los promedios totales de infiltración, antes y después de la vendimia durante la realización de los experimentos con el permeámetro de Guelph. 


\subsection{Valores de infiltración, $\mathrm{Kf}_{s}$ y potencial del flujo matricial}

\subsubsection{Promedios totales de infiltración, $\mathrm{Kf}$ y potencial del flujo matricial a lo largo de la parcela para los viñedos jóvenes y viejos}

Se presentan los valores numéricos de los resultados obtenidos tras calcular los promedios totales de infiltración, $\mathrm{Kf}_{\mathrm{s}}$ y potencial del flujo matricial, junto con las condiciones ambientales del área de estudio (Tabla 3).

Tabla 3. Valores de los promedios totales de infiltración, $K f_{s}$ y potencial del flujo matricial entre los suelos de los viñedos jóvenes y viejos.

\begin{tabular}{|c|c|c|c|c|c|c|c|}
\hline & Loc. & Pend. $\left({ }^{\circ}\right)$ & MO (\%) & $\mathrm{w}(\%)$ & $\begin{array}{c}\text { Inf. } \\
\left(\mathbf{m m ~ h} \mathbf{h}^{-1}\right)\end{array}$ & $\begin{array}{c}\mathbf{K f}_{\mathrm{s}} \\
\left(\mathrm{mm} \mathrm{h}^{-1}\right)\end{array}$ & $\begin{array}{c}\Phi \\
\left(\mathbf{m m}^{2} \mathbf{h}^{-1}\right)\end{array}$ \\
\hline \multirow{3}{*}{ Jóvenes } & Alta & $21,7 \pm 3,7$ & $7,5 \pm 1,7$ & $8,3 \pm 7$ & $103,7(129,3)$ & $15,5(19,6)$ & $14,1(24)$ \\
\hline & Media & $25 \pm 3,8$ & $13,1 \pm 3,7$ & $8,2 \pm 6,3$ & $398,5(400,4)$ & 89,2 (168) & $62,8(23,5)$ \\
\hline & Baja & $19,1 \pm 3,4$ & $5,1 \pm 2,2$ & $5,8 \pm 5,4$ & $236,1(384,9)$ & $25,6(62,6)$ & $35,6(12,1)$ \\
\hline \multirow{3}{*}{ Viejos } & Alta & $18,4 \pm 3,8$ & $7,1 \pm 1,1$ & $10,9 \pm 6,7$ & $42,5(44,7)$ & $13,2(8,3)$ & $5,5(3,1)$ \\
\hline & Media & $22,4 \pm 2,9$ & $6,5 \pm 0,9$ & $10,3 \pm 5,3$ & $20,1(16,2)$ & $6,2(8,9)$ & $2,9(2,2)$ \\
\hline & Baja & $18 \pm 3,1$ & $5,4 \pm 1$ & $9,9 \pm 4,9$ & $16,8(27,4)$ & $5,4(21,8)$ & $2,5(4,1)$ \\
\hline
\end{tabular}

Loc.: localización; Pend.: pendiente; MO: materia orgánica; w: humedad del suelo; Inf. $\left(\mathrm{mm} \mathrm{h}^{-1}\right)$ : tasa de infiltración; $\mathrm{Kf}_{\mathrm{s}}\left(\mathrm{mm} \mathrm{h}^{-1}\right)$ : conductividad hidráulica saturada del suelo; $\Phi\left(\mathrm{mm}^{2} \mathrm{~h}^{-1}\right)$ : potencial del flujo matricial; (): desviación típica estándar.

Los valores generales de la pendiente oscilaron entre $18 \pm 3,1^{\circ}$ y $25 \pm 3,8^{\circ}$, con un mayor grado de inclinación en la parte media. Los datos de materia orgánica fueron elevados, registrándose los mayores porcentajes en las viñas jóvenes en la parte media $(13,1 \pm 3,7 \%)$ y un descenso desde la parte superior hacia la más baja en las antiguas (desde $7,1 \pm 1,1 \%$ a $5,4 \pm 1 \%$ ). El contenido de humedad fue uno de los datos con mayor variabilidad durante el estudio, habiendo un descenso de este parámetro desde la parte superior hacia la inferior.

A primera vista, los resultados de las tasas de infiltración, conductividad hidráulica y potencial del flujo matricial mostraron una elevada desviación típica en cada uno de los promedios, lo que da a entender la elevada disparidad de resultados obtenidos. En general, todos los parámetros analizados fueron mucho más elevados en los viñedos jóvenes que en los viejos. Además, los comportamientos hidrológicos no fueron iguales. En las viñas jóvenes los tres parámetros analizados mostraron sus mayores valores en la parte media de la parcela $\left(398,5 \mathrm{~mm} \mathrm{~h}^{-1}\right.$ de tasas de infiltración, 89,2 $\mathrm{mm} \mathrm{h}^{-1}$ de conductividad hidráulica y $62,8 \mathrm{~mm}^{2} \mathrm{~h}^{-1}$ de potencial del flujo matricial), coincidiendo con la mayor inclinación y alto contenido de materia orgánica que se mencionaba anteriormente. Las cifras más bajas fueron registradas en la parte alta. 
En las viñas viejas se apreció un descenso de la infiltración desde la parte superior a la inferior (desde 42,5 a 16,8 $\mathrm{mm} \mathrm{h}^{-1}$ ). Por último, cabe destacar que los resultados de la conductividad hidráulica y el potencial del flujo matricial marcaron también la misma dinámica hidrológica: descenso de los parámetros conforme se desciende en la parcela $\left(13,2\right.$ a $5,4 \mathrm{~mm} \mathrm{~h}^{-1}$ y 5,5 a $\left.2,5 \mathrm{~mm}^{2} \mathrm{~h}^{-1}\right)$.

\subsubsection{Promedios totales de infiltración, $\mathrm{Kf}_{s}$ y potencial del flujo matricial a lo largo de la parcela para los viñedos jóvenes antes y después de la vendimia}

En la Tabla 4 se presentan los resultados obtenidos para los viñedos jóvenes durante la época previa y posterior a la vendimia. Los valores de materia orgánica no descendieron demasiado entre los experimentos previos y posteriores a la vendimia. Sin embargo, no ocurrió lo mismo con la humedad, donde se pasó de valores inferiores al $4 \%$ a superiores al $11 \%$. Con respecto a los cálculos de las tasas de infiltración, se pudo observar cómo antes de la vendimia se dio un descenso muy drástico desde la parte alta hacia la baja (desde 128,3 $\mathrm{mm} \mathrm{h}^{-1}$ a $13,7 \mathrm{~mm} \mathrm{~h}^{-1}$ ). Por el contrario, tras la vendimia, los valores mayores se dieron en la parte media $\left(388,2 \mathrm{~mm} \mathrm{~h}^{-1}\right)$ coincidiendo con los contenidos más elevados en arenas, materia orgánica y humedad; a continuación en la parte baja $\left(179,6 \mathrm{~mm} \mathrm{~h}^{-1}\right) \mathrm{y}$, por último, los más bajos en la parte alta $\left(70,9 \mathrm{~mm} \mathrm{~h}^{-1}\right)$. Con respecto a los valores de conductividad hidráulica y potencial del flujo matricial, se registró la misma dinámica hidrológica del suelo en ambos contextos espacio-temporales.

Tabla 4. Valores de los promedios de infiltración, Kfs y potencial del flujo matricial antes y después de la vendimia en los suelos de los viñedos jóvenes.

\begin{tabular}{|c|c|c|c|c|c|c|c|}
\hline & Loc. & Pend. $\left({ }^{\circ}\right)$ & MO (\%) & w $(\%)$ & $\begin{array}{c}\text { Inf. } \\
\left(\mathrm{mm} \mathrm{h}^{-1}\right)\end{array}$ & $\begin{array}{c}\mathbf{K f}_{\mathrm{s}} \\
\left(\mathrm{mm} \mathrm{h}^{-1}\right)\end{array}$ & $\begin{array}{c}\Phi \\
\left(\mathrm{mm}^{2} \mathbf{h}^{-1}\right)\end{array}$ \\
\hline \multirow{3}{*}{ Antes } & Alta & $19,5 \pm 2,4$ & $7,2 \pm 0,1$ & $2,8 \pm 2,1$ & $128,3(171,7)$ & $11,1(11,5)$ & $17,3(27)$ \\
\hline & Media & $22,3 \pm 1,3$ & $13,7 \pm 0,2$ & $3,7 \pm 2,7$ & $90(57,3)$ & $30,4(17,5)$ & $51,6(74)$ \\
\hline & Baja & $19,3 \pm 4,6$ & $5,1 \pm 0,2$ & $1,7 \pm 1$ & $13,7(5)$ & $4,3(1,7)$ & $43,5(82,9)$ \\
\hline \multirow{3}{*}{ Después } & Alta & $24,7 \pm 3,2$ & $7,8 \pm 0,4$ & $15,5 \pm 1,6$ & $70,9(55,7)$ & $21,4(16,9)$ & $9,9(7,8)$ \\
\hline & Media & $28,7 \pm 2,5$ & $12,3 \pm 0,3$ & $14,2 \pm 3,4$ & $388,2(187,2)$ & $167,6(133,1)$ & $77,6(61,6)$ \\
\hline & Baja & $19 \pm 2$ & $5,0 \pm 0,2$ & $11,4 \pm 2,4$ & $179,6(130,3)$ & $54,0(39,7)$ & $25(18,4)$ \\
\hline
\end{tabular}

Loc.: localización; Pend.: pendiente; MO: materia orgánica; w: humedad del suelo; Inf. $\left(\mathrm{mm} \mathrm{h}^{-1}\right)$ : tasa de infiltración; $\mathrm{Kf}_{\mathrm{s}}\left(\mathrm{mm} \mathrm{h}^{-1}\right)$ : conductividad hidráulica saturada del suelo; $\Phi\left(\mathrm{mm}^{2} \mathrm{~h}^{-1}\right)$ : potencial del flujo matricial; (): desviación típica estándar.

4.3.3. Promedios totales de infiltración, $\mathrm{Kf}_{s}$ y potencial del flujo matricial a lo largo de las parcelas para los viñedos viejos antes y después de la vendimia

Por último, se presentan los datos referidos al comportamiento hidrológico del suelo en la ladera cultivada con viñedos viejos antes y después de la vendimia (Tabla 5). 
Tabla 5. Valores de los promedios de infiltración, $K f_{s}$ y potencial del flujo matricial antes y después de la vendimia en los suelos de los viñedos viejos.

\begin{tabular}{|c|c|c|c|c|c|c|c|}
\hline & Loc. & Pend. $\left({ }^{\mathbf{(}}\right)$ & MO $(\boldsymbol{\%})$ & $\mathbf{w}(\boldsymbol{\%})$ & $\begin{array}{c}\mathbf{I n f} . \\
\left(\mathbf{m m ~ h}^{-\mathbf{1}}\right)\end{array}$ & $\begin{array}{c}\mathbf{K f}_{\mathbf{s}} \\
\left(\mathbf{m m ~ h}^{-\mathbf{1}}\right)\end{array}$ & $\begin{array}{c}\boldsymbol{\Phi} \\
\left(\mathbf{m m}^{\mathbf{2}} \mathbf{h}^{-\mathbf{}}\right)\end{array}$ \\
\hline \multirow{3}{*}{ Antes } & Alta & $18 \pm 0,8$ & $7,9 \pm 0,2$ & $6,5 \pm 4,6$ & $24(10)$ & $8,1(3,5)$ & $3,8(1,6)$ \\
\cline { 2 - 8 } & Media & $20,3 \pm 1,3$ & $7 \pm 0,2$ & $7,3 \pm 5,3$ & $19,4(15,8)$ & $6,2(5,2)$ & $2,9(2,4)$ \\
\cline { 2 - 8 } & Baja & $16,5 \pm 1,3$ & $6,0 \pm 0,2$ & $6,5 \pm 3,3$ & $24,7(36)$ & $8,0(12,1)$ & $3,6(5,6)$ \\
\hline \multirow{3}{*}{ Después } & Alta & $18,8 \pm 6,5$ & $6,0 \pm 0,1$ & $16,7 \pm 3,5$ & $67,2(65)$ & $19,9(19,3)$ & $7,9(9,4)$ \\
\cline { 2 - 8 } & Media & $25,3 \pm 0,6$ & $5,7 \pm 0,2$ & $14,2 \pm 1,3$ & $21,4(23,6)$ & $6,1(6,7)$ & $2,8(3,1)$ \\
\cline { 2 - 8 } & Baja & $20 \pm 4$ & $4,5 \pm 0,1$ & $14,4 \pm 1,1$ & $6,3(3,8)$ & $1,9(1,1)$ & $0,9(0,5)$ \\
\hline
\end{tabular}

Loc.: localización; Pend.: pendiente; MO: materia orgánica; w: humedad del suelo; Inf. $\left(\mathrm{mm} \mathrm{h}^{-1}\right)$ : tasa de infiltración; $\mathrm{Kf}_{\mathrm{s}}\left(\mathrm{mm} \mathrm{h}^{-1}\right)$ : conductividad hidráulica saturada del suelo; $\Phi\left(\mathrm{mm}^{2} \mathrm{~h}^{-1}\right)$ : potencial del flujo matricial; (): desviación típica estándar.

La humedad fue la propiedad más variable antes y después de la vendimia, incrementándose en casi un $10 \%$. La materia orgánica fue algo inferior también.

Por lo que respecta a los resultados finales, las tasas de infiltración mostraron un incremento sustancial después de la vendimia. En los momentos anteriores a la recolección de la uva, los valores fueron muy próximos entre sí desde la parte alta a la baja, tanto en las cifras de infiltración (24 mm h-1 $19,4 \mathrm{~mm} \mathrm{~h}^{-1}$ y $\left.24,7 \mathrm{~mm} \mathrm{~h}^{-1}\right)$, como en las de conductividad hidráulica $\left(8,1 \mathrm{~mm} \mathrm{~h}^{-1}, 6,2 \mathrm{~mm} \mathrm{~h}^{-1}\right.$ y $\left.6,2 \mathrm{~mm} \mathrm{~h}^{-1}\right)$ y el potencial del flujo matricial $\left(3,8 \mathrm{~mm}^{2} \mathrm{~h}^{-1}, 2,9 \mathrm{~mm}^{2} \mathrm{~h}^{-1}\right.$ y $\left.3,6 \mathrm{~mm}^{2} \mathrm{~h}^{-1}\right)$. Se apreció un ligero descenso de los valores en la parte media de la parcela.

Tras la vendimia, coincidiendo con un decrecimiento de contenido en materia orgánica y un aumento en la humedad del suelo, las tasas de infiltración fueron más elevadas en la parte alta y media de la parcela $\left(67,2 \mathrm{~mm} \mathrm{~h}^{-1}\right.$ y $\left.21,4 \mathrm{~mm} \mathrm{~h}^{-1}\right)$ frente a la parte baja $\left(6,3 \mathrm{~mm} \mathrm{~h}^{-1}\right)$. Como ya ocurriera con los resultados de los promedios generales, se puede observar cómo los resultados de conductividad hidráulica y potencial matricial muestran la misma dinámica hidrológica.

\subsection{Factores ambientales condicionantes de la variación de la infiltración}

Se ha elaborado una serie de gráficos de puntos con los datos de humedad, materia orgánica, pendientes y tasas de infiltración, y calculado su grado de determinación (Fig. 6) para comprobar el grado de relación entre los factores ambientales (variables independientes) y los resultados de las tasas de infiltración (variables dependientes) en los diferentes puntos de las parcelas (partes alta, media y baja). Se observó que antes de la vendimia en los viñedos jóvenes solo el grado de humedad tuvo un nivel de relación medio $\left(\mathrm{R}^{2}=0,5\right)$ con la infiltración. A medida que los valores de humedad aumentaban, las tasas también lo hacían. Por otra parte, se observa cómo en la parcela con cepas viejas la pendiente y la humedad mostraron un grado de determinación muy elevado, $\mathrm{R}^{2}=0,92$ y $\mathrm{R}^{2}=0,98$, respectivamente. 

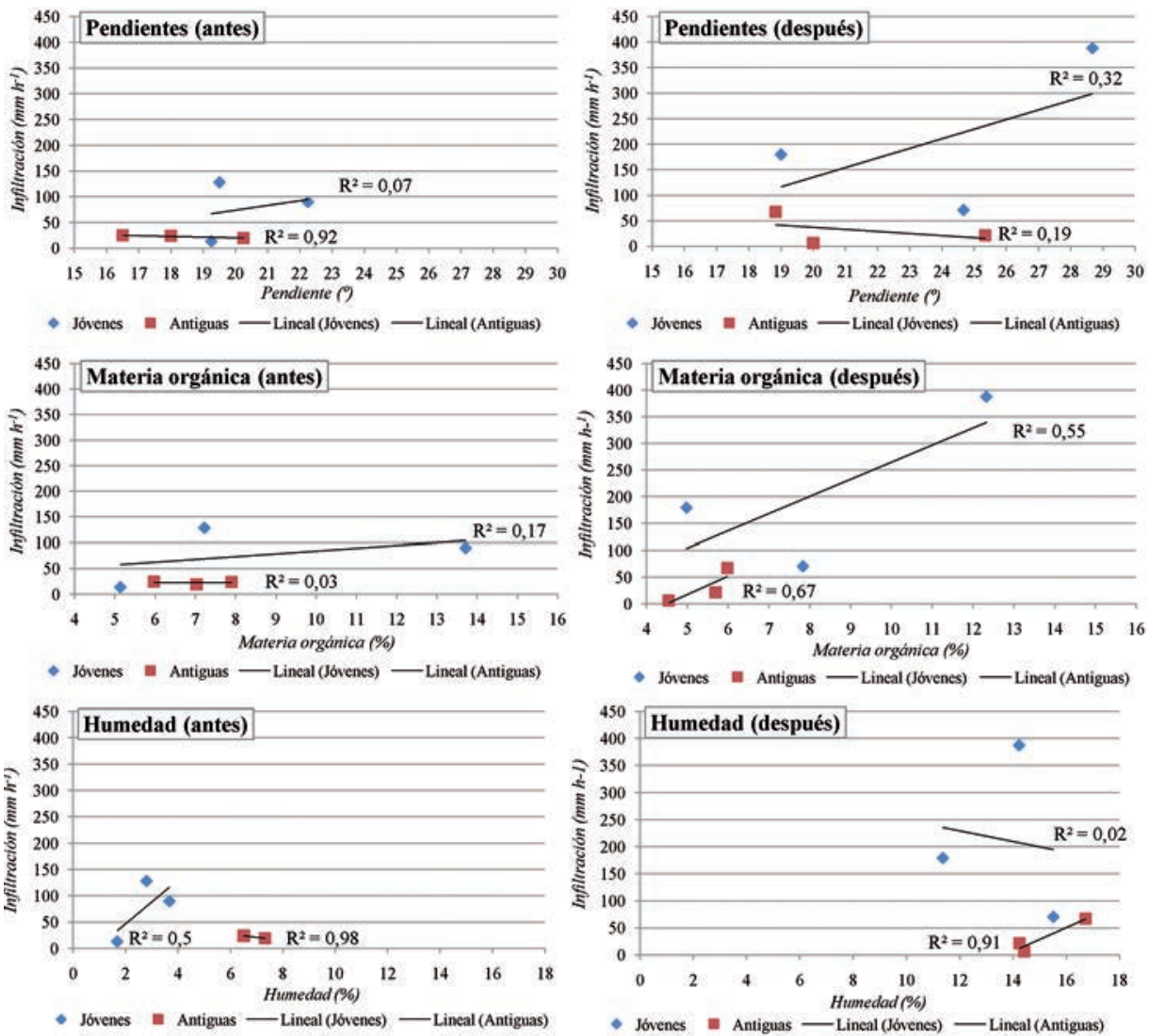

Figura 6. Relación entre las características ambientales y las tasas de infiltración, antes y después de la vendimia.

Tras la vendimia, con el descenso de la materia orgánica se apreció un aumento de la determinación con las tasas de infiltración en los viñedos jóvenes $\left(\mathrm{R}^{2}=0,55\right)$. Por su parte, el aumento de la humedad y el descenso de la materia orgánica en este período muestran elevados grados de determinación con las tasas de infiltración $\left(\mathrm{R}^{2}=0,91\right.$ y $\left.\mathrm{R}^{2}=0,67\right)$.

\section{Discusión}

Las mediciones de la intensidad de los procesos hidrológicos en forma de escorrentía superficial e infiltración en los viñedos europeos se ha convertido en un tema de gran relevancia para la ciencia del suelo y hoy sigue vigente en las nuevas publicaciones por las posibles consecuencias negativas para la sostenibilidad de la vid (Novara et al., 2011; Biddoccu et al., 2013; Lieskovský y Kenderessy, 2014; Galati et al., 2015; Rodrigo Comino y Senciales González, 2015). 
Los perfiles y análisis de suelos descritos en ambas parcelas con viñedos de diferente edad a lo largo de una geoforma principal (ladera) han mostrado una sucesión de tipos de horizontes similares: i) un Ap superficial entremezclado con el mantillo y; ii) un $\mathrm{B} / \mathrm{C}$ muy removido y compactado por la maquinaria. Al mismo tiempo, también se observa una clara pérdida de profundidad y empobrecimiento de los horizontes más superficiales desde la parte alta de cada parcela a la inferior. Sin embargo, Rodrigo Comino et al., (2015b) para la misma área de estudio, confirmaron a través de marcas botánicas sobre el callo del tocón de las vides que las diferencias entre éstas y el actual nivel del suelo eran mayores en la parte superior que en la inferior, donde predominaba la acumulación. A priori, existiría una contradicción entre ambos resultados si el mecanismo principal de activación de la erosión fuera, como en la mayoría de los viñedos mediterráneos, la escorrentía superficial (Kosmas et al., 1997; Ramos et al., 2015; Prosdocimi et al., 2016a). No obstante, como ya comprobaron Rodrigo Comino et al., (2015a, 2016) los procesos de infiltración son muy elevados debido a la alta pedregosidad (superior al $60 \%$ en numerosos puntos), la elevada pendiente y, el mayor contenido en arenas en superficie y de arcillas en profundidad, lo que conlleva a que aparezcan mecanismos de escorrentía sub-superficial (Hewlett y Hibbert, 1967; Gupta et al., 1993, 2006; Ramos et al., 2000; MacDonald et al., 2012; Archer et al., 2013; Bodner et al., 2013; Nasri et al., 2015).

El agua tras infiltrarse se desplaza a lo largo de la ladera desde la parte superior a la inferior. Sin embargo, para el caso concreto de las parcelas analizadas, las discontinuidades provocadas por las prácticas agrícolas (compactación, destrucción de agregados o remoción de los primeros $20 \mathrm{~cm}$ de espesor del suelo) y las diferencias pedológicas intra-parcelarias per se, generaron que los resultados obtenidos marcaran irregularidades en esta dinámica hidrológica, rompiendo la linealidad del proceso.

Los cálculos obtenidos de infiltración, conductividad hidráulica y potencial del flujo matricial a través del permeámetro de Guelph a lo largo de las dos parcelas y en distintas épocas del año han manifestado claramente la alteración de la dinámica hidrológica anteriormente formulada. En primer lugar, en los viñedos jóvenes, coincidiendo con la época anterior a la vendimia en primavera-verano, con un mayor contenido en materia orgánica y menor humedad en el suelo las tasas de infiltración, conductividad hidráulica y el potencial del flujo matricial fueron más elevadas desde la parte superior a la inferior. Esto mostró que la dinámica de la escorrentía sub-superficial dentro de un mecanismo mixto (escorrentía superficial y sub-superficial) siguiera su mecanismo natural (Hewlett y Hibbert, 1967; Imeson y Lavee, 1998). Sin embargo, tras la vendimia estos suelos con una mayor pedregosidad, grado de inclinación, todavía sin una potente cobertura radicular que pueda sujetar el suelo firmemente y los claros signos de compactación por el pisoteo y el paso de la maquinaria, vieron alterado el comportamiento hidrológico del suelo, siendo mayor la infiltración en la parte media de la parcela, que en la superior.

Por su parte, en los viñedos viejos antes de la vendimia, se apreció una homogeneidad en las tasas de infiltración (algo menores en la parte media de la ladera). No obstante, tras la cosecha se observó un aumento de las ratios desde arriba hacia abajo, aunque con una elevada variabilidad en los resultados obtenidos (mayores desviaciones típicas). 
Esta elevada variabilidad de los procesos hidrológicos por causas antropogénicas y naturales con matices según el área de estudio (diversos tipos de suelos, ambientes climáticos distintos y prácticas agrícolas diferentes) ha sido confirmada en numerosos estudios en viñedos de España (Ortigosa Izquierdo y Lasanta Martínez, 1984; Martínez Murillo y Ruiz Sinoga, 2003; Arnáez et al., 2007; Casalí et al., 2009; Ramos et al., 2000; Rodrigo Comino y Senciales González, 2015; Prosdocimi et al., 2016b), Francia (Blavet et al., 2009; Paroissien et al., 2010; Follain et al., 2012; Chevigny et al., 2014; Quiquerez et al., 2014) o Italia (Biddoccu et al., 2013; Galati et al., 2015; Prosdocimi et al., 2016a).

Esta alteración de la dinámica hidrológica y el incremento de la escorrentía sub-superficial puede implicar graves problemas de transporte de solutos a lo largo de ladera, lavado de nutrientes, pérdida de suelo, deslizamientos de ladera por piping o generación de regueros y cárcavas, podredumbre de las raíces de las vides de la parte inferior de la parcela y, por consiguiente empobrecimiento y pérdida de productividad (Richter, 1979, 1980; Poesen et al., 1997; Ramos y Martínez-Casasnovas, 2006; Bruggisser et al., 2010; De Baets et al., 2011; Martínez-Murillo et al., 2013; Novara et al., 2013; Morvan et al., 2014; Ramos et al., 2015; Rodrigo Comino et al., 2015a; Galati et al., 2015).

Con respecto a la metodología utilizada, podemos confirmar la utilidad del permeámetro de Guelph para analizar la variabilidad de los procesos hidrológicos en los suelos de las viñas. Sin embargo, cabe destacar que dicho instrumento, no ha sido utilizado con regularidad en viñedos hasta la fecha, pese a estar bastante extendido su uso para fines hidrogeológicos y agronómicos desde hace décadas (Reynolds, 1986; Elrick y Reynolds, 1992; Xiang, 1994; Bagarello, 1997; Kodešová et al., 2010; Ronayne et al., 2012; Reynolds y Lewis, 2012; Nasri et al., 2015).

No obstante, una de las mayores críticas que se puede hacer a este método y que se extrae tras la lectura de dichas investigaciones es que no se puede encontrar un método estándar de su aplicación, en lo que se refiere a tiempo de duración del experimento, número de repeticiones o, simplemente, en qué unidades se debe expresar, dificultando enormemente que los datos puedan ser comparados entre regiones o tipos de usos. Por ejemplo, es común que se realicen diferentes cálculos a la hora de decidir qué cifra utilizar para el steady rate o constante de infiltración en las ecuaciones. Algunos autores se decantan por seleccionar el valor que se repite entre tres y cuatro ocasiones durante la medición y otros cuando el promedio no supera un coeficiente de variación del 10\%. En nuestro caso, hemos considerado realizar el promedio de todos los valores obtenidos, con objeto de utilizar siempre el mismo criterio de selección y tener en cuenta la respuesta del suelo durante todo el experimento.

Otro aspecto metodológico relevante observado es que los valores de infiltración y los de conductividad hidráulica a lo largo del estudio, al aplicarse las distintas ecuaciones, han obtenido cifras muy dispares. La inclusión de un factor o constante relacionado con la macrocospicidad del suelo (Reynolds, 1986; Elrick y Reynolds, 1992; Xiang, 1994) provoca la obtención de resultados mucho más bajos que los de la tasa de infiltración calculada directamente teniendo en cuenta el tamaño del orificio y el área de la 
base del permeámetro. Así, para cada una de las interpretaciones de las cifras absolutas se debe tener en cuenta que los resultados provienen de ecuaciones distintas.

De esta forma, queremos resaltar la necesidad de una estandarización del método, tanto en su utilización directa en campo como en el análisis de los datos posteriores. A partir de esta investigación, se pretende sentar las bases de una futura metodología utilizando esta herramienta, al menos para una aplicación en el estudio de las variaciones hidrológicas del suelo en campos cultivados con vides u otras aplicaciones relacionadas con la ciencia del suelo o la Geografía Física.

\section{Conclusiones}

El presente estudio ha mostrado la alta variabilidad y alteración de los procesos hidrológicos de ladera en relación con la influencia de dos factores: naturales y antropogénicos. Los naturales estarían relacionados con los cambios en el contenido de materia orgánica, humedad del suelo, textura, posición en la ladera y grado de inclinación de la pendiente, y los condicionados por el ser humano permanecerían unidos fundamentalmente a las prácticas agrícolas.

Los resultados obtenidos tras el análisis y la descripción de perfiles de suelos han mostrado un suelo con textura franco limosa, con mayor cantidad de partículas de arenas en superficie y de arcillas en profundidad, muy pedregosos, con elevado contenido en materia orgánica, no muy profundos $(<30 \mathrm{~cm}) \mathrm{y}$, con horizontes Ap marcados y, B/C removidos y moderadamente compactados.

Con el uso de un permeámetro de Guelph, se han detectado unas tasas de infiltración elevadas que, junto con las anteriores características edáficas analizadas, responden a procesos hidrológicos ligados a escorrentía sub-superficial con una dirección marcada desde la parte superior de la parcela a la inferior. Sin embargo, dicha tendencia queda alterada y la variabilidad aumenta considerablemente tras la época de la vendimia y el laboreo del suelo utilizando maquinaria pesada.

Las elevadas tasas de infiltración, la generación de escorrentía sub-superficial y la alteración de los mecanismos naturales de escorrentía superficial, junto con el incremento de la variabilidad hidrológica intra-parcelaria, puede desencadenar procesos erosivos que favorezcan la pérdida de suelo, nutrientes y fertilidad a medio-largo plazo.

\section{Agradecimientos}

En primer lugar, nuestro agradecimiento a la cooperativa de Weinbauverband Mosel, y las bodegas de Gebrüder Steffes (Waldrach) y Langguth (Traben-Trarbach) por facilitar el área de estudio. También queremos agradecer a los tres revisores sus correcciones y sugerencias. Gracias también al editor, el profesor José M. García Ruiz por mostrar interés en el articulo y estar pendiente en todo momento del proceso. Finalmente, agradecemos a Caixa-Bank y al Servicio de Intercambio Académico Alemán (DAAD) por la beca concedida a Jesús Rodrigo-Comino para realizar este trabajo. 


\section{Referencias}

Archer, N.A.L., Bonell, M., Coles, N., MacDonald, A.M., Auton, C.A., Stevenson, R. 2013. Soil characteristics and landcover relationships on soil hydraulic conductivity at a hillslope scale: A view towards local flood management. Journal of Hydrology 497, 208-222. Doi: 10.1016/j. jhydrol.2013.05.043.

Arnáez, J., Lasanta, T., Ruiz-Flaño, P., Ortigosa, L. 2007. Factors affecting runoff and erosion under simulated rainfall in Mediterranean vineyards. Soil \& Tillage Research 93, 324-334. Doi: 10.1016/j.still.2006.05.013.

Bagarello, V., 1997. Influence of well preparation on field-saturated hydraulic conductivity measured with the Guelph Permeameter. Geoderma 80, 169-180. Doi: 10.1016/S00167061(97)00051-7.

Bagarello, V., Castellini, M., Di Prima, S., Iovino, M. 2014. Soil hydraulic properties determined by infiltration experiments and different heights of water pouring. Geoderma 213, 492-501. Doi: 10.1016/j.geoderma.2013.08.032.

Biddoccu, M., Ferraris, S., Cavallo, E., Opsi, F., Previati, M., Canone, D. 2013. Hillslope Vineyard Rainfall-Runoff Measurements in Relation to Soil Infiltration and Water Content. Procedia Environmental Sciences 19, 351-360. Doi: 10.1016/j.proenv.2013.06.040.

Blavet, D., De Noni, G., Le Bissonnais, Y., Leonard, M., Maillo, L., Laurent, J.Y., Asseline, J., Leprun, J.C., Arshad, M.A., Roose, E. 2009. Effect of land use and management on the early stages of soil water erosion in French Mediterranean vineyards. Soil \& Tillage Research 106, 124-136. Doi: 10.1016/j.still.2009.04.010.

Bodner, G., Scholl, P., Loiskandl, W., Kaul, H.P. 2013. Environmental and management influences on temporal variability of near saturated soil hydraulic properties. Geoderma 204-205, 120 129. Doi: 10.1016/j.geoderma.2013.04.015.

Bruggisser, O.T., Schmidt-Entling, M.H., Bacher, S. 2010. Effects of vineyard management on biodiversity at three trophic levels. Biological Conservation 143, 1521-1528. Doi: 10.1016/j. biocon.2010.03.034.

Cadot, Y., Caillé, S., Thiollet-Scholtus, M., Samson, A., Barbeau, G., Cheynier, V. 2012. Characterisation of typicality for wines related to terroir by conceptual and by perceptual representations. An application to red wines from the Loire Valley. Food Quality and Preference 24, 48-58. Doi: 10.1016/j.foodqual.2011.08.012.

Casalí, J., Giménez, R., De Santisteban, L., Álvarez-Mozos, J., Mena, J., Del Valle de Lersundi, J. 2009. Determination of long-term erosion rates in vineyards of Navarre (Spain) using botanical benchmarks. Catena 78, 12-19. Doi: 10.1016/j.catena.2009.02.015.

Cerdà, A., 1997. Soil erosion after land abandonment in a semiarid environment of southeastern Spain. Arid Soil Research 11, 163-176. Doi: 10.1080/15324989709381469.

Chevigny, E., Quiquerez, A., Petit, C., Curmi, P. 2014. Lithology, landscape structure and management practice changes: Key factors patterning vineyard soil erosion at metre-scale spatial resolution. Catena 121, 354-364. Doi: 10.1016/j.catena.2014.05.022.

Corbane, C., Jacob, F., Raclot, D., Albergel, J., Andrieux, P. 2012. Multitemporal analysis of hydrological soil surface characteristics using aerial photos: A case study on a Mediterranean vineyard. Int. J. Appl. Earth Obs. Geoinformation 18, 356-367. Doi: 10.1016/j. jag.2012.03.009.

Costantini, E.A.C., Agnelli, A.E., Fabiani, A., Gagnarli, E., Mocali, S., Priori, S., Simoni, S., Valboa, G. 2015. Short-term recovery of soil physical, chemical, micro- and mesobiological functions in a new vineyard under organic farming. SOIL 1, 443-457. Doi: 10.5194/soil-1443-2015. 
Davies, B.E. 1974. Loss-on-Ignition as an Estimate of Soil Organic Matter. Soil Science Society of America Journal 38. Doi: 10.2136/sssaj1974.03615995003800010046x.

De Baets, S., Poesen, J., Meersmans, J., Serlet, L. 2011. Cover crops and their erosion-reducing effects during concentrated flow erosion. Catena 85, 237-244. Doi: 10.1016/j.catena. 2011.01.009.

Elrick, D.E., Reynolds, W.D. 1992. Methods for analyzing constant-head well Permeameter data. Soil Science Society of America Journal 56, 320-323. Doi: 10.2136/sssaj1992.03615995005600010052x.

Fischer, U., Roth, D., Christmann, M. 1999. The impact of geographic origin, vintage and wine estate on sensory properties of Vitis vinifera $\mathrm{cv}$. Riesling wines. Food Quality and Preference 10 (4-5), 281-288. Doi: 10.1016/S0950-3293(99)00008-7.

Follain, S., Ciampalini, R., Crabit, A., Coulouma, G., Garnier, F. 2012. Effects of redistribution processes on rock fragment variability within a vineyard topsoil in Mediterranean France. Geomorphology 175-176, 45-53. Doi: 10.1016/j.geomorph.2012.06.017.

Galati, A., Gristina, L., Crescimanno, M., Barone, E., Novara, A. 2015. Towards More Efficient Incentives for Agri-environment Measures in Degraded and Eroded Vineyards. Land Degradation \& Development 26, 557-564. Doi: 10.1002/ldr.2389.

Gruber, B., Kosegarten, H., 2002. Depressed growth of non-chlorotic vine grown in calcareous soil is an iron deficiency symptom prior to leaf chlorosis. Journal of Plant Nutrition and Soil Science 165, 111-117. Doi: 10.1002/1522-2624(200202)165:1<111::AID-JPLN111>3.0.CO;2-B.

Gupta, R.K., Rudra, R.P., Dickinson, W.T., Patni, N.K., Wall, G.J. 1993. Comparison of saturated hydraulic conductivity measured by various field methods. Transactions of the ASAE 36, 51-55. Doi: 10.13031/2013.28313.

Gupta, R.K., Rudra, R.P., Parkin, G. 2006. Analysis of spatial variability of hydraulic conductivity at field scale. Canadian Biosystems Engineering 48, 1.55-1.62.

Gwenzi, W., Hinz, C., Holmes, K., Phillips, I.R., Mullins, I.J. 2011. Field-scale spatial variability of saturated hydraulic conductivity on a recently constructed artificial ecosystem. Geoderma 166, 43-56. Doi: 10.1016/j.geoderma.2011.06.010.

Hewlett, J.D., Hibbert, A.R. 1967. Factors affecting the response of small watersheds to precipitation in humid areas. En Sopper, W.E., Lull, H.W. (eds.), Classics in Physical Geography Revisited, Pergamon Press, New York, pp. 275-290.

Huang, M., Rodger, H., Barbour, S.L. 2014. An evaluation of air permeability measurements to characterize the saturated hydraulic conductivity of soil reclamation covers. Canadian Journal of Soil Science 95, 15-26. Doi: 10.4141/cjss-2014-072.

Huang, M., Zettl, J.D., Lee Barbour, S., Pratt, D. 2016. Characterizing the spatial variability of the hydraulic conductivity of reclamation soils using air permeability. Geoderma 262, 285-293. Doi: 10.1016/j.geoderma.2015.08.014.

Imeson, A.C., Lavee, H., 1998. Soil erosion and climate change: the transect approach and the influence of scale. Geomorphology 23, 219-227. Doi: 10.1016/S0169-555X(98)00005-1.

IUSS Working Group WRB 2014. World Reference Base for Soil Resources 2014. World Soil Resources Report. FAO, Roma.

IUSS Working Group WRB 2007. Land Evaluation. Towards a revised framework. 2nd ed., Land and Water discussion paper, FAO, Roma.

IUSS Working Group WRB 2006. Guidelines for constructing smallscale map legends using the WRB. 2nd ed., World Soil Resources. FAO, Roma.

Jačka, L., Pavlásek, J., Kuráž, V., Pech, P. 2014. A comparison of three measuring methods for estimating the saturated hydraulic conductivity in the shallow subsurface layer of mountain podzols. Geoderma 219-220, 82-88. Doi: 10.1016/j.geoderma.2013.12.027.

Jackson, R.S. 2014. Wine science. Principles and Applications. Fourth Edition, Elsevier, London, 984 pp. 
Jirků, V., Kodešová, R., Nikodem, A., Mühlhanselová, M., Žigová, A. 2013. Temporal variability of structure and hydraulic properties of topsoil of three soil types. Geoderma 204-205, 43 58. Doi: 10.1016/j.geoderma.2013.03.024.

Kodešová, R., Šimůnek, J., Nikodem, A., Jirků, V. 2010. Estimation of the Dual-Permeability Model Parameters using Tension Disk Infiltrometer and Guelph Permeameter. Vadose Zone Journal 9. Doi: 10.2136/vzj2009.0069.

Köpppen, W., Geiger, R. 1954. Klima der Erde. Justus Perthes Ed., Darmstadt.

Kosmas, C., Danalatos, N., Cammeraat, L.H., Chabart, M., Diamantopoulos, J., Farand, R., Gutiérrez, L., Jacob, A., Marqués, H., Martínez-Fernández, J., Mizara, A., Moustakas, N., Nicolau, J.M., Oliveros, C., Pinna, G., Puddu, R., Puigdefabregas, J., Roxo, M., Simao, A., Stamou, G., Tomasi, N., Usai, D., Vacca, A. 1997. The effect of land use on runoff and soil erosion rates under Mediterranean conditions. Catena 29, 45-59. Doi: 10.1016/S03418162(96)00062-8.

Kumar, S., Sekhar, M., Reddy, D.V., Mohan Kumar, M.S. 2010. Estimation of soil hydraulic properties and their uncertainty: comparison between laboratory and field experiment. Hydrological Processes 24, 3426-3435. Doi: 10.1002/hyp.7775.

Leonard, J., Andrieux, P. 1998. Infiltration characteristics of soils in Mediterranean vineyards in Southern France. Catena 32, 209-223. Doi: 10.1016/S0341-8162(98)00049-6.

Lesch, S.M., Corwin, D.L. 2003. Using the dual-pathway parallel conductance model to determine how different soil properties influence conductivity survey data. Agronomy Journal 95, 365379. Doi: 10.2134/agronj2003.3650.

Lieskovský, J., Kenderessy, P. 2014. Modelling the effect of vegetation cover and different tillage practices on soil erosion in vineyards: a case study in Vráble (Slovakia) using WATEM/ SEDEM. Land Degradation \& Development 25, 288-296. Doi: 10.1002/ldr.2162.

Likar, M., Vogel-Mikuš, K., Potisek, M., Hančević, K., Radić, T., Nečemer, M., Regvar, M. 2015. Importance of soil and vineyard management in the determination of grapevine mineral composition. Science of the Total Environment 505, 724-731. Doi: 10.1016/ j.scitotenv.2014.10.057.

López-Piñeiro, A., Muñoz, A., Zamora, E., Ramírez, M. 2013. Influence of the management regime and phenological state of the vines on the physicochemical properties and the seasonal fluctuations of the microorganisms in a vineyard soil under semi-arid conditions. Soil \& Tillage Research 126, 119-126. Doi: 10.1016/j.still.2012.09.007.

MacDonald, A.M., Maurice, L., Dobbs, M.R., Reeves, H.J., Auton, C.A. 2012. Relating in situ hydraulic conductivity, particle size and relative density of superficial deposits in a heterogeneous catchment. Journal of Hydrology 434-435, 130-141. Doi: 10.1016/j.jhydrol.2012.01.018.

Martínez-Casasnovas, J.A., Ramos, M.C., García-Hernández, D. 2009. Effects of land-use changes in vegetation cover and sidewall erosion in a gully head of the Penedès region (northeast Spain). Earth Surface Processes and Landforms 34, 1927-1937. Doi: 10.1002/esp.1870.

Martínez-Murillo, J.F., Nadal-Romero, E., Regüés, D., Cerdà, A., Poesen, J. 2013. Soil erosion and hydrology of the western Mediterranean badlands throughout rainfall simulation experiments: A review. Catena 106, 101-112. Doi: 10.1016/j.catena.2012.06.001.

Martínez-Murillo, J.F., Ruiz-Sinoga, J.D. 2003. Incidencia de algunas propiedades físicas de suelos en su respuesta hidrológica ante diferentes usos bajo condiciones mediterráneas (Montes de Málaga). Edafología 10, 57-62.

Morvan, X., Naisse, C., Malam Issa, O., Desprats, J.F., Combaud, A., Cerdan, O. 2014. Effect of ground-cover type on surface runoff and subsequent soil erosion in Champagne vineyards in France. Soil Use and Management 30, 372-381. Doi: 10.1111/sum.12129. 
Nasri, B., Fouché, O., Torri, D. 2015. Coupling published pedotransfer functions for the estimation of bulk density and saturated hydraulic conductivity in stony soils. Catena 131, 99-108. Doi: 10.1016/j.catena.2015.03.018.

Novara, A., Gristina, L., Guaitoli, F., Santoro, A., Cerdà, A. 2013. Managing soil nitrate with cover crops and buffer strips in Sicilian vineyards. Solid Earth 4, 255-262. Doi: 10.5194/ se-4-255-2013.

Novara, A., Gristina, L., Saladino, S.S., Santoro, A., Cerdà, A. 2011. Soil erosion assessment on tillage and alternative soil managements in a Sicilian vineyard. Soil \& Tillage Research 117, 140-147. Doi: 10.1016/j.still.2011.09.007.

Ortigosa Izquierdo, L.M., Lasanta Martínez, T. 1984. El papel de la escorrentía en la organización textural de suelos cultivados en pendiente: modelos en viñedos de La Rioja. Cuadernos de Investigación Geográfica 9, 99-111.

Paroissien, J.B., Lagacherie, P., Le Bissonnais, Y. 2010. A regional-scale study of multi-decennial erosion of vineyard fields using vine-stock unearthing-burying measurements. Catena 82 , 159-168. Doi: 10.1016/j.catena.2010.06.002.

Peter, K.D., Ries, J.B. 2013. Infiltration rates affected by land levelling measures in the Souss valley, South Morocco. Zeitschrift für Geomorphologie 57, 59-72.

Poesen, J., van Wesemael, B., Govers, G., Martínez-Fernández, J., Desmet, P., Vandaele, K., Quine, T., Degraer, G. 1997. Patterns of rock fragment cover generated by tillage erosion. Geomorphology 18, 183-197.

Porta, J., López-Acevedo, M., Poch, R. 2014. Edafología: uso y protección de suelos. $3^{\text {a }}$ ed., Mundiprensa, Madrid.

Price, K., Jackson, C.R., Parker, A.J. 2010. Variation of surficial soil hydraulic properties across land uses in the southern Blue Ridge Mountains, North Carolina, USA. Journal of Hydrology 383 (3-4), 256-268. Doi: 10.1016/j.jhydrol.2009.12.041.

Prosdocimi, M., Cerdà, A., Tarolli, P. 2016a. Soil water erosion on Mediterranean vineyards: A review. Catena 141, 1-21. Doi: 10.1016/j.catena.2016.02.010.

Prosdocimi, M., Jordán, A., Tarolli, P., Keesstra, S., Novara, A., Cerdà, A. 2016b. The immediate effectiveness of barley straw mulch in reducing soil erodibility and surface runoff generation in Mediterranean vineyards. Science of the Total Environment 547, 323-330. Doi: 10.1016/j. scitotenv.2015.12.076.

Quiquerez, A., Chevigny, E., Allemand, P., Curmi, P., Petit, C., Grandjean, P. 2014. Assessing the impact of soil surface characteristics on vineyard erosion from very high spatial resolution aerial images (Côte de Beaune, Burgundy, France). Catena 116, 163-172. Doi: 10.1016/j. catena.2013.12.002.

Ramos, M.C., Benito, C., Martínez-Casasnovas, J.A. 2015. Simulating soil conservation measures to control soil and nutrient losses in a small, vineyard dominated, basin. Agriculture, Ecosystems, Environment 213, 194-208. Doi: http://dx.doi.org/10.1016/j.agee.2015.08.004.

Ramos, M.C., Martínez-Casasnovas, J.A. 2006. Impact of land levelling on soil moisture and runoff variability in vineyards under different rainfall distributions in a Mediterranean climate and its influence on crop productivity. Journal of Hydrology 321, 131-146. Doi: 10.1016/j.jhydrol.2005.07.055.

Ramos, M.C., Nacci, S., Pla, I. 2000. Soil sealing and its influence on erosion rates for some soils in the Mediterranean area. Soil Science 165, 398-403.

Resolution OIV/VITI 333/2010, 2010. Definition of vitivinicultural "Terroir". T. Asamblea General del OIV, Tbilisi, Georgia.

Reynolds, W.D. 1986. The Guelph Permeameter method for in situ measurement of field-saturated hydraulic conductivity and matric flux potential. Unpublished $\mathrm{PhD}$, Guelph University, Guelph, Ontario, Canadá. 
Reynolds, W.D., Elrick, D.E. 2002. Constant head well permeameter (vadose zone). En J.H. Dane, G.C. Topp (eds.), Methods of Soil Analysis, Physical Methods, Soil Science Society of America, Inc., Madison, WI (USA), pp. 844-858.

Reynolds, W.D., Lewis, J.K. 2012. A drive point application of the Guelph Permeameter method for coarse-textured soils. Geoderma 187-188, 59-66. Doi: 10.1016/j.geoderma.2012.04.004.

Richter, G. 1980. On the Soil Erosion Problem in the Temperate Humid Area of Central Europe. GeoJournal 4, 279-287.

Richter, G. 1979. Bodenerosion in Rebanlagen des Moselgebietes. Ergebnisse quantitativer Untersuchungen 1974-1977. Forschungsstelle Bodenerosion d. Univ. Trier, Trier.

Richter, G. 1975. Der Aufbau der Forschungsstelle Bodenerosion und die ersten Messungen in Weinbergslagen. Forschungsstelle Bodenerosion d. Universitat Trier, Trier.

Rienzner, M., Gandolfi, C. 2014. Investigation of spatial and temporal variability of saturated soil hydraulic conductivity at the field-scale. Soil \& Tillage Research 135, 28-40. Doi: http:// dx.doi.org/10.1016/j.still.2013.08.012.

Rodrigo-Comino, J., Brings, C., Lassu, T., Iserloh, T., Senciales, J., Martínez-Murillo, J., Ruiz-Sinoga, J., Seeger, M., Ries, J. 2015a. Rainfall and human activity impacts on soil losses and rill erosion in vineyards (Ruwer Valley, Germany). Solid Earth 6, 823-837. Doi: $10.5194 / \mathrm{se}-6-823-2015$.

Rodrigo-Comino, J., Lassu, T., González, J.M.S., Ruiz-Sinoga, J.D.R., Seeger, K.M., Ries, J.B. 2015b. Estudio de procesos geomorfodinámicos en campos cultivados de viñedos sobre laderas en pendientes en el valle del Ruwer (Alemania). Cuadernos Geográficos 54, 6-26.

Rodrigo-Comino, J., Iserloh, T., Morvan, X., Malam Issa, O., Naisse, C., Keesstra, S.D., Cerdà, A., Prosdocimi, M., Arnáez, J., Lasanta, T., Ramos, M.C., Marqués, M.J., Ruiz Colmenero, M., Bienes, R., Ruiz-Sinoga, J.D., Seeger, M., Ries, J.B. 2016. Soil Erosion Processes in European Vineyards: A Qualitative Comparison of Rainfall Simulation Measurements in Germany, Spain and France. Hydrology 3, 1-19. Doi: 10.3390/hydrology3010006.

Rodrigo-Comino, J., Senciales González, J.M., 2015. Ratio LE para el ajuste de perfiles longitudinales en cursos fluviales de montaña. Aplicación a la cuenca del río Almáchar (Málaga, España). Cuaternario y Geomorfología 29, 31-56.

Ronayne, M.J., Houghton, T.B., Stednick, J.D. 2012. Field characterization of hydraulic conductivity in a heterogeneous alpine glacial till. Journal of Hydrology 458-459, 103-109. Doi: 10.1016/j. jhydrol.2012.06.036.

Rosell, R.A., Gasparoni, J.C., Galantini, J.A. 2001. Soil organic matter evaluation. En R. Lal, J. Kimble, R. Follet, B. Stewart (eds.), Assessment Methods for Soil Carbon, Lewis Publishers, USA, pp. 311-322.

Ruiz-Sinoga, J.D., Martínez-Murillo, J.F. 2009. Effects of soil surface components on soil hydrological behaviour in a dry Mediterranean environment (Southern Spain). Geomorphology 108, 234-245. Doi: 10.1016/j.geomorph.2009.01.012.

Salome, C., Coll, P., Lardo, E., Villenave, C., Blanchart, E., Hinsinger, P., Marsden, C., Le Cadre, E. 2014. Relevance of use-invariant soil properties to assess soil quality of vulnerable ecosystems: The case of Mediterranean vineyards. Ecological Indicators 43, 83-93. Doi: 10.1016/j.ecolind.2014.02.016.

Soilmoisture Equipment Corp. 2008. Model 2800K1 Guelph Permeameter Operating Instructions. Soilmoisture Equipment Corp., Santa Barbara, CA.

Taguas, E.V., Guzmán, E., Guzmán, G., Vamwalleghem, T., Gómez, J.A. 2015. Characteristics and importance of rill and gully erosion: a case study in a small catchment of a marginal olive grove. Cuadernos de Investigatión Geográfica 41 (1), 107-126. Doi: 10.18172/cig.2644.

Taylor, J.A., Coulouma, G., Lagacherie, P., Tisseyre, B. 2009. Mapping soil units within a vineyard using statistics associated with high-resolution apparent soil electrical conductivity data and factorial discriminant analysis. Geoderma 153, 278-284. Doi: 10.1016/j.geoderma.2009.08.014. 
Van Leeuwen, C., Bois, B., De Resseguier, L., Roby, J.P. 2010. New methods and technologies to describe the environment in terroir studies. En VIII International Terroir Congress, Consiglio per la Ricerca e la Sperimentazione in Agricoltura, Centro di Ricerca per la Viticoltura, Soave, Italia, pp. 2-13.

Wu, L., Swan, J.B., Paulson, W.H., Randall, G.W. 1992. Tillage effects on measured soil hydraulic properties. Soil \& Tillage Research 25, 17-33. Doi: 10.1016/0167-1987(92)90059-K.

Xiang, J. 1994. Improvements in evaluating constant-head permeameter test data. Journal of Hydrology 162, 77-97. Doi: 10.1016/0022-1694(94)90005-1.

Zhang, Z.F., Groenevelt, P.H., Parkin, G.W. 1998. The well-shape factor for the measurement of soil hydraulic properties using the Guelph Permeameter. Soil \& Tillage Research 49, 219-221. Doi: 10.1016/S0167-1987(98)00174-3. 